\title{
Carbonylative Ring Opening of Terminal Epoxides at Atmospheric Pressure
}

\author{
Scott E. Denmark* and Moballigh Ahmad \\ Department of Chemistry, Roger Adams Laboratory, University of Illinois, Urbana, Illinois \\ 61801
}

\section{SUPPORTING INFORMATION}

Table of Contents

page

General Experimental

Dicobalt Octacarbonyl Catalyzed Carbonylation of 1a-p.

References

NMR Spectra

$S 26$

GC/SFC Traces

$S 43$

\section{General Experimental}

All carbonylation reactions (except high pressure reactions) were performed in oven $\left(150{ }^{\circ} \mathrm{C}\right)$ and/or flame dried Schlenk flasks. High-pressure reactions (41 bar CO) were performed in a custom built six-cell stainless steal autoclave. THF, $\mathrm{CH}_{2} \mathrm{Cl}_{2}$ were dried by percolation through a column packed with neutral alumina and a column packed with Q5 reactant, a supported copper catalyst for scavenging oxygen, under a positive pressure of argon. Methanol was freshly distilled from $\mathrm{Mg}(\mathrm{OMe})_{2}$ prior used. All terminal epoxides were used as received. Propylene oxide and cyclohexene oxide were heated to reflux over calcium hydride for $12 \mathrm{~h}$, then were distilled, and stored in Schlenk flasks.

${ }^{1} \mathrm{H}$ NMR, and ${ }^{13} \mathrm{C}$ NMR spectra were recorded at $400 \mathrm{MHz}$ or $500 \mathrm{MHz}$. Spectra are 
referenced to residual chloroform $\left(\delta 7.26 \mathrm{ppm},{ }^{1} \mathrm{H} ; \delta 77.0 \mathrm{ppm},{ }^{13} \mathrm{C}\right)$. Chemical shifts are reported in ppm. Multiplicities are indicated by s (singlet), d (doublet), t (triplet), q (quartet), $\mathrm{m}$ (multiplet), hept (heptet), and br (broad). Coupling constants $J$, are reported in Hertz. Lowresolution electron impact (EI) mass spectra were obtained with a typical ionization voltage of 70 eV. Chemical ionization (CI) spectra were obtained using methane as the carrier gas. Data are reported in the form of $(\mathrm{m} / \mathrm{z})$. Infrared spectra (IR) are reported in $\mathrm{cm}^{-1}$ with indicated relative intensities: s (strong, 67-100\%); m (medium, 34-66\%); w (weak, 0-33\%). Melting point (mp) was determined in sealed tubes and are corrected. Analytical thin-layer chromatography was performed on Merck silica gel 60 with F-254 indicator (pre coated plates). Visualization was accomplished with $p$-anisaldehyde stain. Gas chromatography (GC) was performed using a flame ionization detector and a trifluoroacetyl $\gamma$-cyclodextrin (G-TA, $30 \times 0.25 \mathrm{~mm}$, manufactured by CHIRALDEX $\left.^{\mathrm{TM}}\right)$. The separations were performed with a $\mathrm{H}_{2}$ head pressure of 12 psi. Injector temperature was $250{ }^{\circ} \mathrm{C}$; the detector temperature was $300{ }^{\circ} \mathrm{C}$. Analytical supercritical fluid chromatography (SFC) was performed on packed-column SFC built-in photometric detector $(\lambda=$ 220, $258 \mathrm{~nm})$ using Daiciel Chiralpak OD column. Retention times $\left(t_{\mathrm{R}}\right)$ and peak areas for SFC and GC were obtained from reporting integrators.

Epoxides 1a, 1b, 1c, 1f, 1g, 1h, 1i, 1l, 1p were obtained from commercial vendors and used as received. Epoxides $\mathbf{1 d},{ }^{1} \mathbf{1 e},{ }^{2} \mathbf{1 j},{ }^{3} \mathbf{1 k},{ }^{4} \mathbf{1} \mathbf{m}^{5} \mathbf{1 n}^{6}$ and $\mathbf{1 0}{ }^{7}$ were synthesized according to literature procedures. Carbon monoxide was received from Matheson TRI.GAS, C.P grade $(\mathrm{CP}=$ Chemically pure), lot number 4005200303. Dicobalt octacarbonyl was purchased from Strem Chemicals and was sublimed in $100 \mathrm{~mL}$ glass sublimation chamber at $0.5 \mathrm{mmHg}$ pressure using acetone/dry ice cold finger at room temperature to $35^{\circ} \mathrm{C}$. 


\section{Experimental Procedures}

\section{General Procedure I. Dicobalt Octacarbonyl Catalyzed Carbonylation of 1a-p.}

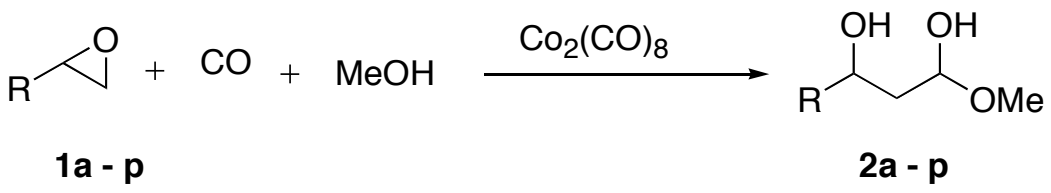

In a glove box, $\mathrm{Co}_{2}(\mathrm{CO})_{8}(84.5 \mathrm{mg}, 0.25 \mathrm{mmol}$. 0.05 equiv. $)$ was placed in a $100-\mathrm{mL}$ Schlenk flask equipped with a magnetic stir bar. The flask was sealed with a rubber septum and was removed from a dry box. The flask was purged three times for 2 sec. with carbon monoxide (filled in $12.7 \mathrm{~cm}$ latex balloon). Freshly distilled methanol $(5.0 \mathrm{~mL})$ was added and the mixture was stirred for $2 \mathrm{~min}$. before the epoxides $(5 \mathrm{mmol}$ ) was added in one portion. The mixture was allowed to stir at room temperature for $24 \mathrm{~h}$. After the given reaction time, the carbon monoxide balloon was removed and carefully vented in the hood. The reaction mixture was diluted with ether $(50 \mathrm{~mL})$ and was stirred for $30-60$ minutes to precipitate the cobalt complex. The mixture was passed through silica gel $(20 \mathrm{X} 100 \mathrm{~mm})$, using diethyl ether as the eluent and upon careful removal of solvent in vacuo provided the products, that can be further purified (if necessary) by flash chromatography on silica gel using hexane/ethyl acetate as the eluent.

\section{Preparation of Methyl 3-Hydroxybutanoate (2a) [Table 2, Entry 1]}

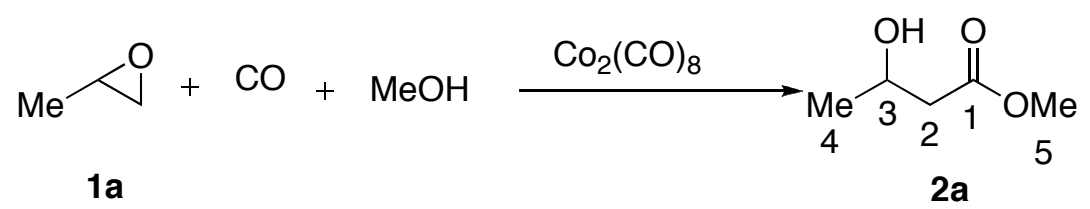

Following General Procedure I, $\mathrm{Co}_{2}(\mathrm{CO})_{8}(84.5 \mathrm{mg}, 0.25 \mathrm{mmol}, 0.05$ equiv. $)$, freshly distilled methanol $(5.0 \mathrm{~mL})$ and $\mathbf{1 a}(350 \mu \mathrm{L}, 5.0 \mathrm{mmol})$ were stirred under a CO atmosphere $(1$ atm) at room temperature for $24 \mathrm{~h}$. The reaction mixture was diluted with diethyl ether (50 $\mathrm{mL})$ 
and was stirred for $30 \mathrm{~min}$, then passed through silica gel $(20 \mathrm{X} 100 \mathrm{~mm})$, with diethyl ether (10 $\mathrm{mL}$ ) as the eluent. Careful removal of solvent under reduced pressure afforded $525 \mathrm{mg}(89 \%)$ of 2a as clear colorless oil. NMR spectral data matched that previously reported in the literature. ${ }^{8}$

Data for 2a:

${ }^{1} \mathrm{H} \mathrm{NMR} \quad\left(500 \mathrm{MHz}, \mathrm{CDCl}_{3}\right)$

4.19 (m, 1 H), 3.70 (s, 3 H), 2.96 (d, 1 H), 2.49 (dd, $J=16.3,3.4,1$ H), 2.42 (dd, $J=16.3,8.7,1 \mathrm{H}), 1.22(\mathrm{~d}, 3 \mathrm{H})$

${ }^{13} \mathrm{C} \mathrm{NMR} \quad\left(125 \mathrm{MHz}, \mathrm{CDCl}_{3}\right)$

$173.3,64.2,51.7,42.5,22.4$

$\underline{\text { IR }}$ (neat)

3422 (s), 2975 (s), 2930 (s), 2093 (w), 1724 (s), 1439 (m), 1374 (m), 1299 (m), $1261(\mathrm{~m}), 1176(\mathrm{~m}), 1124(\mathrm{~m}), 1088(\mathrm{~m}), 1072(\mathrm{~m}), 1006(\mathrm{~m}), 946(\mathrm{~m}), 851(\mathrm{w})$

Preparation of Methyl 3-Hydroxyheptanoate (2b) [Table 2, Entry 2]
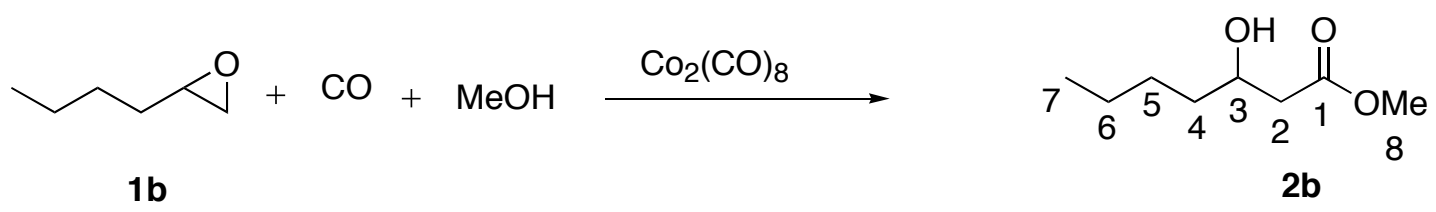

Following General Procedure $\mathrm{I}, \mathrm{Co}_{2}(\mathrm{CO})_{8}(84.5 \mathrm{mg}, 0.25 \mathrm{mmol}, 0.05$ equiv.), freshly distilled methanol $(5.0 \mathrm{~mL})$ and $\mathbf{1 b}(600 \mu \mathrm{L}, 5.0 \mathrm{mmol})$ were stirred under a $\mathrm{CO}$ atmosphere (1 atm) at room temperature for $24 \mathrm{~h}$. The reaction mixture was diluted with diethyl ether (50 mL) and was stirred for $30 \mathrm{~min}$, then passed through silica gel $(20 \mathrm{X} 100 \mathrm{~mm})$, with diethyl ether (10 $\mathrm{mL}$ ) as the eluent. Careful removal of solvent in vacuo followed by column chromatography $\left(\mathrm{SiO}_{2}, 20 \mathrm{~mm} \mathrm{X} 20 \mathrm{~cm}\right.$, hexane/EtOAc, hexane, 20/1, 3/1) to afforded $710 \mathrm{mg}(89 \%)$ of $\mathbf{2 b}$ as clear colorless oil. NMR spectral data matched that previously reported in the literature. ${ }^{9}$ 


\section{Data for 2b:}

${ }^{1} \mathrm{H} \mathrm{NMR} \quad\left(500 \mathrm{MHz}, \mathrm{CDCl}_{3}\right)$

$4.00(\mathrm{~m}, 1 \mathrm{H}), 3.71(\mathrm{~s}, 3 \mathrm{H}), 2.86(\mathrm{~d}, 1 \mathrm{H}), 2.51(\mathrm{dd}, J=16.4,3.0,1 \mathrm{H}), 2.40(\mathrm{dd}, J$

$=16.4,9.1,1 \mathrm{H}), 1.29-1.53($ br m, $6 \mathrm{H}) 0.90(\mathrm{t}, 3 \mathrm{H})$

${ }^{13} \mathrm{C} \mathrm{NMR} \quad\left(125 \mathrm{MHz}, \mathrm{CDCl}_{3}\right)$

$173.4,67.9,51.7,41.1,36.2,27.6,22.5,13.9$

$\underline{\text { IR }}$

(neat)

3446 (w), 2956 (m), 2932 (m), 2861 (w), 1738 (s), 1438 (m), $1291(\mathrm{w}), 1169(\mathrm{~m})$, $1125(w), 1040(w)$

\section{Preparation of Methyl 3-Hydroxy-6-heptenoate (2c) [Table 2, Entry 3]}
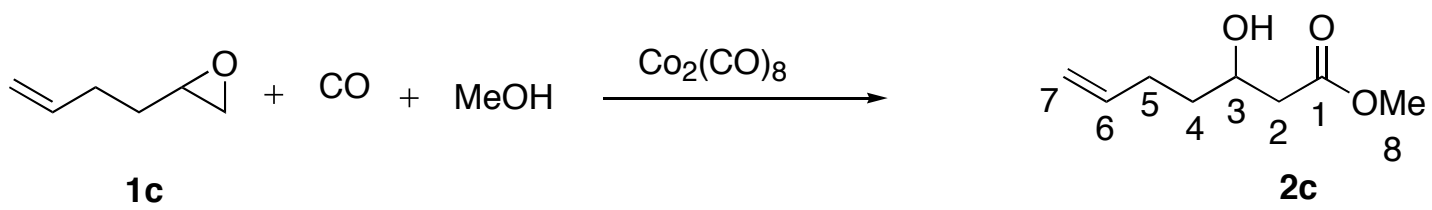

Following General Procedure I, $\mathrm{Co}_{2}(\mathrm{CO})_{8}(84.5 \mathrm{mg}, 0.25 \mathrm{mmol}, 0.05$ equiv. $)$, freshly distilled methanol $(5.0 \mathrm{~mL})$ and $1 \mathrm{c}(564 \mu \mathrm{L}, 5.0 \mathrm{mmol})$ were stirred under a CO atmosphere $(1$ atm) at room temperature for $24 \mathrm{~h}$. The reaction mixture was diluted with diethyl ether $(50 \mathrm{~mL})$ and was stirred for $30 \mathrm{~min}$., then passed through silica gel (20 X $100 \mathrm{~mm})$, with diethyl ether (10 $\mathrm{mL}$ ) as the eluent. Careful removal of solvent in vacuo followed by column chromatography $\left(\mathrm{SiO}_{2}, 20 \mathrm{~mm} \mathrm{X} 20 \mathrm{~cm}\right.$, hexane/EtOAc, hexane, 20/1, 3/1) to afforded $760 \mathrm{mg}(96 \%)$ of 2c as clear colorless oil. NMR spectral data matched that previously reported in the literature. ${ }^{10}$ Data for 2c:

${ }^{1} \mathrm{H} \mathrm{NMR} \quad\left(500 \mathrm{MHz}, \mathrm{CDCl}_{3}\right)$

$5.82(\mathrm{~m}, 1 \mathrm{H}), 5.05(\mathrm{dq}, 17.2,1.7,1 \mathrm{H}), 4.98(\mathrm{dq}, 9.7,1.4,1 \mathrm{H}), 4.03(\mathrm{~m}, 1 \mathrm{H})$, 
$3.71(\mathrm{~s}, 3 \mathrm{H}), 2.90(\mathrm{~d}, 1 \mathrm{H}), 2.52(\mathrm{dd}, J=16.6,3.1,1 \mathrm{H}), 2.43(\mathrm{dd}, J=16.3,9.0,1$ $\mathrm{H}), 2.18(\mathrm{~m}, 2 \mathrm{H}), 1.58(\mathrm{~m}, 2 \mathrm{H})$

${ }^{13} \mathrm{C}$ NMR $\quad\left(125 \mathrm{MHz}, \mathrm{CDCl}_{3}\right)$

$173.3,137.9,115.0,67.4,51.7,41.1,35.5,29.7$

$\underline{\mathrm{IR}}$ (neat) 3445 (w), 3077 (w), 2923 (w), 2849 (w), 1737 (m), $1641(w), 1438(\mathrm{~m}), 1359(\mathrm{w})$, $1295($ w), $1247(w), 1200($ w), $1168(w), 1121(w), 1082(w), 1062(w), 995(w)$, $912(w)$

Preparation of Methyl 3-Hydroxy-4-phenylbutanoate (2d) [Table 2, Entry 4]
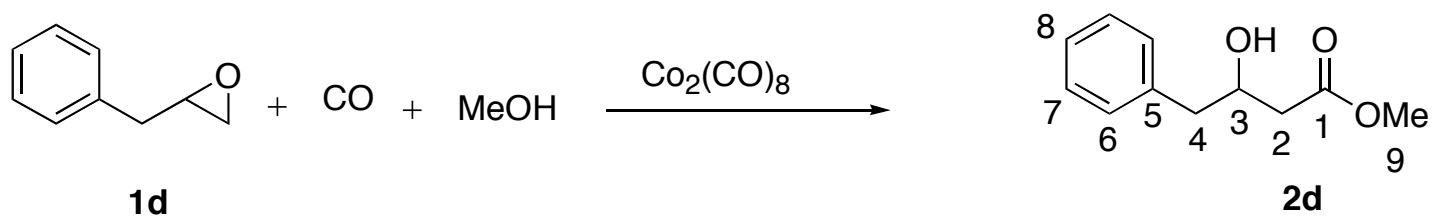

2d

Following General Procedure I, $\mathrm{Co}_{2}(\mathrm{CO})_{8}(84.5 \mathrm{mg}, 0.25 \mathrm{mmol}, 0.05$ equiv.), freshly distilled methanol $(5.0 \mathrm{~mL})$ and $\mathbf{1 d}(657 \mu \mathrm{L}, 5.0 \mathrm{mmol})$ were stirred under a $\mathrm{CO}$ atmosphere $(1$ atm) at room temperature for $24 \mathrm{~h}$. The reaction mixture was diluted with diethyl ether $(50 \mathrm{~mL})$ and was stirred for $30 \mathrm{~min}$, then passed through silica gel (20 X $100 \mathrm{~mm})$, with diethyl ether (10 $\mathrm{mL}$ ) as the eluent. Careful removal of solvent in vacuo followed by column chromatography $\left(\mathrm{SiO}_{2}, 20 \mathrm{~mm}\right.$ X $20 \mathrm{~cm}$, hexane/EtOAc, hexane, 20/1, 3/1) to afforded $529 \mathrm{mg}(54 \%)$ of $\mathbf{2 d}$ as clear colorless oil. NMR spectral data matched that previously reported in the literature. ${ }^{11}$

Data for 2d:

${ }^{1} \mathrm{H} \mathrm{NMR} \quad\left(500 \mathrm{MHz}, \mathrm{CDCl}_{3}\right)$

7.31 (br t, 2 H), 7.21-7.25 (br m, 3 H), 4.27 (m, 1 H), 3.70 (s, 3 H), 2.86 (m, $2 \mathrm{H}$ ), $2.67(\mathrm{dd}, J=13.6,6.1,1 \mathrm{H}), 2.53(\mathrm{dd}, J=16.3,3.4,1 \mathrm{H}), 2.46(\mathrm{dd}, J=16.3,8.8$, 
$1 \mathrm{H}), 1.22(\mathrm{~d}, 3 \mathrm{H})$

${ }^{13} \mathrm{C}$ NMR $\quad\left(125 \mathrm{MHz}, \mathrm{CDCl}_{3}\right)$

$173.0,137.5,129.3,128.5,126.6,69.0,51.7,42.9,40.3$

$\underline{\text { IR }}$ (neat)

3433 (m), 3087 (w), 3063 (w), 3029 (w), 3004 (w), 2953 (s), 2852 (w), 1731 (s), 1604 (m), 1495 (m), 1455 (m), 1439 (m) 1269 (s), 1200 (s), 1150 (s), 1083 (s), 1054 (s), 1030 (s), 995 (s), 854 (s), 749 (s), 701 (s)

Preparation of Methyl 3-Hydroxy-5-phenylpentanoate (2e) [Table 2, Entry 5]
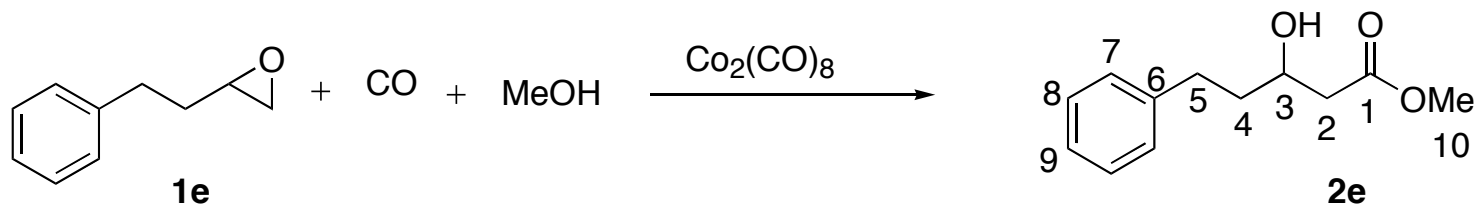

Following General Procedure $\mathrm{I}, \mathrm{Co}_{2}(\mathrm{CO})_{8}(84.5 \mathrm{mg}, 0.25 \mathrm{mmol}, 0.05$ equiv.), freshly distilled methanol $(5.0 \mathrm{~mL})$ and $1 \mathrm{e}(657 \mu \mathrm{L}, 5.0 \mathrm{mmol})$ were stirred under a $\mathrm{CO}$ atmosphere $(1$ atm) at room temperature for $24 \mathrm{~h}$. The reaction mixture was diluted with diethyl ether $(50 \mathrm{~mL})$ and was stirred for $30 \mathrm{~min}$., then passed through silica gel (20 X $100 \mathrm{~mm})$, with diethyl ether (10 $\mathrm{mL}$ ) as the eluent. Careful removal of solvent in vacuo followed by column chromatography $\left(\mathrm{SiO}_{2}, 20 \mathrm{~mm}\right.$ X $20 \mathrm{~cm}$, hexane/EtOAc, hexane, 20/1, 3/1) to afforded $625 \mathrm{mg}(64 \%)$ of $2 \mathrm{e}$ as clear colorless oil. NMR spectral data matched that previously reported in the literature. ${ }^{12}$ Data for 2e:

${ }^{1} \mathrm{H} \mathrm{NMR} \quad\left(500 \mathrm{MHz}, \mathrm{CDCl}_{3}\right)$

7.32 (m, 2 H), 7.25 (br m, 3 H), 4.06 (m, 1 H), 3.75 (s, 3 H), $3 . .05$ (d, 1 H), 2.87

(m, $1 \mathrm{H}), 2.75(\mathrm{~m}, 1 \mathrm{H}), 2.57(\mathrm{dd}, J=16.6,3.4,1 \mathrm{H}), 2.50(\mathrm{dd}, J=16.6,8.7,1 \mathrm{H})$, $2.46(\mathrm{dd}, J=16.3,8.8,1 \mathrm{H}), 1.22(\mathrm{~d}, 3 \mathrm{H}), 1.88(\mathrm{~m}, 1 \mathrm{H}), 1.79(\mathrm{~m}, 1 \mathrm{H})$ 
${ }^{13} \mathrm{C} \mathrm{NMR} \quad\left(125 \mathrm{MHz}, \mathrm{CDCl}_{3}\right)$

$173.3,141.6,128.3,128.5,125.8,67.1,51.7,41.1,38.0,31.7$

$\underline{\text { IR }} \quad$ (neat)

3447 (w), 3085 (w), 3062 (w), 3027 (w), 3002 (w), 3027 (w), 2951 (w), 2861 (w), 1732 (s), 1603 (m), 1496 (m), 1454 (m), 1438 (s), 1302 (s), 1253 (s), 1175 (s), 1155 (s), 1080 (s), 1025 (s), 1002 (s), 936 (s) 749 (s), 700 (s)

\section{Preparation of Methyl 4-Chloro-3-Hydroxybutanoate (2f) [Table 2, Entry 6]}

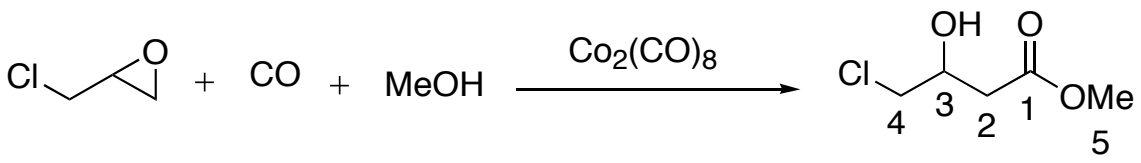

$1 f$

$2 \mathrm{f}$

Following General Procedure I, $\mathrm{Co}_{2}(\mathrm{CO})_{8}(84.5 \mathrm{mg}, 0.25 \mathrm{mmol}, 0.05$ equiv. $)$, freshly distilled methanol $(5.0 \mathrm{~mL})$ and $\mathbf{1 f}(391 \mu \mathrm{L}, 5.0 \mathrm{mmol})$ were stirred under a $\mathrm{CO}$ atmosphere (1 atm) at room temperature for $24 \mathrm{~h}$. The reaction mixture was diluted with diethyl ether $(50 \mathrm{~mL})$ and was stirred for $30 \mathrm{~min}$. in air, then passed through silica gel $(20 \times 100 \mathrm{~mm})$, with diethyl ether $(10 \mathrm{~mL})$ as the eluent. Careful removal of solvent in vacuo followed by column chromatography $\left(\mathrm{SiO}_{2}, 20 \mathrm{~mm}\right.$ X $20 \mathrm{~cm}$, hexane/EtOAc, hexane, 20/1, 3/1) to afforded $556 \mathrm{mg}$ (73\%) of $\mathbf{2 f}$ as clear colorless oil. NMR spectral data matched that previously reported in the literature. $^{13}$

Data for 2f:

ㅁN NMR $\quad\left(500 \mathrm{MHz}, \mathrm{CDCl}_{3}\right)$

$4.26(\mathrm{~m}, 1 \mathrm{H}), 3.73(\mathrm{~s}, 3 \mathrm{H}), 3.61(\mathrm{~m}, 2 \mathrm{H}), 3.05(\mathrm{~d}, J=5.1,1 \mathrm{H}), 2.65(\mathrm{~m}, 2 \mathrm{H})$, $1.22(\mathrm{~d}, 3 \mathrm{H})$ 
${ }^{13} \mathrm{C} \mathrm{NMR} \quad\left(125 \mathrm{MHz}, \mathrm{CDCl}_{3}\right)$

$172.1,67.9,52.0,48.1,38.2$

$\underline{\mathrm{IR}}$ (neat)

$3446(\mathrm{~m}), 2956(\mathrm{w}), 2359(\mathrm{w}), 1734$ (s), 1437 (m), $1370(\mathrm{w}), 1309(\mathrm{w}), 1266(\mathrm{w})$, $1199(w), 1152(w), 1090(w), 1057(w), 961(w), 926(w), 853(w), 759(w)$

Preparation of Methyl 4-Benzyloxy-3-Hydroxybutanoate (2g) [Table 2, Entry 7]

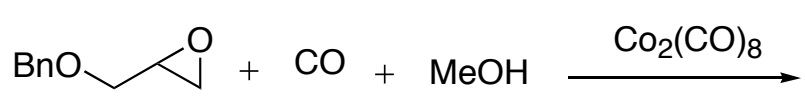

$1 \mathrm{~g}$

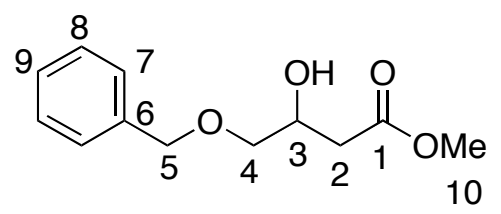

$2 g$

Following General Procedure I, $\mathrm{Co}_{2}(\mathrm{CO})_{8}(84.5 \mathrm{mg}, 0.25 \mathrm{mmol}, 0.05$ equiv. $)$, freshly distilled methanol $(5.0 \mathrm{~mL})$ and $1 \mathrm{~g}(762 \mu \mathrm{L}, 5.0 \mathrm{mmol})$ were stirred under a CO atmosphere $(1$ atm) at room temperature for $24 \mathrm{~h}$. The reaction mixture was diluted with diethyl ether $(50 \mathrm{~mL})$ and was stirred for $30 \mathrm{~min}$ in air, then passed through silica gel (20 X $100 \mathrm{~mm})$, with diethyl ether $(10 \mathrm{~mL})$ as the eluent. Careful removal of solvent in vacuo followed by column chromatography $\left(\mathrm{SiO}_{2}, 20 \mathrm{~mm}\right.$ X $20 \mathrm{~cm}$, hexane/EtOAc, hexane, 20/1, 3/1) to afforded $935 \mathrm{mg}$ (83\%) of $\mathbf{2 g}$ as clear colorless oil. NMR spectral data matched that previously reported in the literature. $^{14}$

Data for 2g:

${ }^{1} \mathrm{H} \mathrm{NMR} \quad\left(500 \mathrm{MHz}, \mathrm{CDCl}_{3}\right)$

7.29-7.36 (br m, $5 \mathrm{H}), 4.56$ (s, $2 \mathrm{H}), 4.24$ (m, $1 \mathrm{H}), 3.69$ (s, $3 \mathrm{H}), 3.52$ (dd, $J=9.5$, $4.3,1 \mathrm{H}), 3.47(\mathrm{dd}, J=9.6,6.0,1 \mathrm{H}), 2.93(\mathrm{~d}, J=5.3,1 \mathrm{H}), 2.55(\mathrm{~d}, J=6.6,2 \mathrm{H})$ 
${ }^{13} \mathrm{C} \mathrm{NMR} \quad\left(125 \mathrm{MHz}, \mathrm{CDCl}_{3}\right)$

$172.4,137.7,128.3,127.7,127.6,73.3,73.0,67.1,51.7,38.0$

$\underline{\mathrm{IR}}$ (neat)

$3453(\mathrm{~m}), 3030$ (w), 2946 (w), 2931 (w), 2863 (w), 1737 (s), $1496(\mathrm{w}), 1453$ (m), $1438(\mathrm{~m}), 1361(\mathrm{~m}), 1255(\mathrm{w}), 1206(\mathrm{w}), 1170(\mathrm{~m}), 1102(\mathrm{~m}), 1005(\mathrm{w}), 739(\mathrm{~m})$, $699(\mathrm{~m})$

\section{Preparation of Methyl 4-Phenoxy-3-Hydroxybutanoate (2h) [Table 2, Entry 8]}
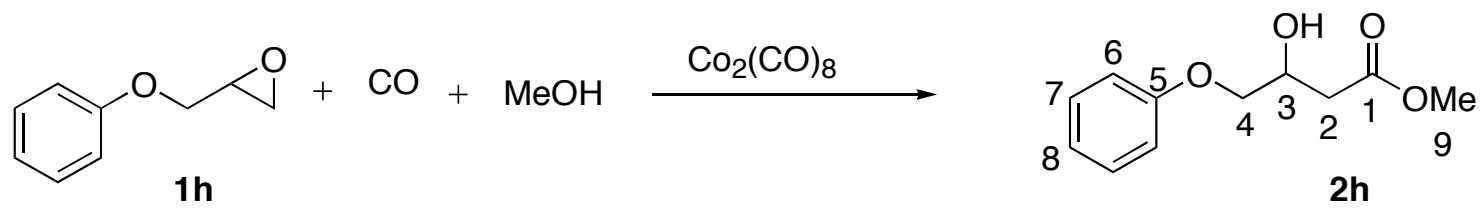

Following General Procedure I, $\mathrm{Co}_{2}(\mathrm{CO})_{8}(84.5 \mathrm{mg}, 0.25 \mathrm{mmol}, 0.05$ equiv. $)$, freshly distilled methanol $(5.0 \mathrm{~mL})$ and $\mathbf{1 h}(678 \mu \mathrm{L}, 5.0 \mathrm{mmol})$ were stirred under a CO atmosphere (1 atm) at room temperature for $24 \mathrm{~h}$. The reaction mixture was diluted with diethyl ether (50 $\mathrm{mL})$ and was stirred for $30 \mathrm{~min}$ in air, then passed through silica gel (20 X $100 \mathrm{~mm})$, with diethyl ether $(10 \mathrm{~mL})$ as the eluent. Careful removal of solvent in vacuo followed by column chromatography $\left(\mathrm{SiO}_{2}, 20 \mathrm{~mm}\right.$ X $20 \mathrm{~cm}$, hexane/EtOAc, hexane, 20/1, 3/1) to afforded $903 \mathrm{mg}$ $(86 \%)$ of $\mathbf{2 h}$ as white solid.

Data for 2h:

Mp $\quad 51-52^{\circ} \mathrm{C}$

${ }^{1} \mathrm{H} \mathrm{NMR} \quad\left(500 \mathrm{MHz}, \mathrm{CDCl}_{3}\right)$

7.27 (br t, $J=8.05,2 \mathrm{H}, \mathrm{HC}(7)), 6.96$ (br t, $J=7.3,1 \mathrm{H}, \mathrm{HC}(8)), 6.90$ (d, $J=7.8$, $2 \mathrm{H}, \mathrm{HC}(6)), 4.42(\mathrm{~m}, 1 \mathrm{H}, \mathrm{HC}(3)), 3.97$ (d, $J=5.1,2 \mathrm{H}, \mathrm{HC}(4)), 3.71(\mathrm{~s}, 3 \mathrm{H}$, $\mathrm{HC}(9)), 3.40(\mathrm{~d}, J=4.8,1 \mathrm{H}, \mathrm{OH}) 2.67$ (m, $2 \mathrm{H}, \mathrm{HC}(2))$ 
${ }^{13} \mathrm{C} \mathrm{NMR} \quad\left(125 \mathrm{MHz}, \mathrm{CDCl}_{3}\right)$

$172.2(\mathrm{C}(1)), 158.2(\mathrm{C}(5)), 129.3(\mathrm{C}(7)), 120.9(\mathrm{C}(8)), 114.3(\mathrm{C}(6)), 70.5(\mathrm{C}(4))$, $66.5(\mathrm{C}(3)), 51.7(\mathrm{C}(9)), 37.8(\mathrm{C}(2))$

$\underline{\mathrm{IR}}$ (neat) $3450(\mathrm{w}), 2952(\mathrm{w}), 2868(\mathrm{w}), 1735(\mathrm{~s}), 1599(\mathrm{~m}), 1587(\mathrm{~m}), 1497(\mathrm{~m}), 1454(\mathrm{w})$, $1438(\mathrm{~m}), 1364(\mathrm{w}), 1292(\mathrm{~m}), 1245(\mathrm{~s}), 1172(\mathrm{~m}), 1078(\mathrm{w}), 1043(\mathrm{~m}), 1000(\mathrm{w})$, $925(w), 884(w), 814(w), 755(m), 692(m)$

$\underline{\mathrm{MS}} \quad(\mathrm{CI}, 70 \mathrm{eV})$

210 (3), 193 (5), 179 (37), 161 (96), 147 (8), 133 (57), 119 (100), 107 (23), 95 (28), 85 (25), 71 (17), 59 (26)

TLC $\quad R f 0.20$ (hexane/EtOAc, 4/1) [silica gel, p-anisaldehyde stain]

Analysis $\quad \mathrm{C}_{11} \mathrm{H}_{14} \mathrm{O}_{4}(210.23)$

Calculated: $\quad$ C, $62.85 ; \quad \mathrm{H}, 6.71 \%$

Found: $\quad$ C, $63.19 ; \quad$ H, $6.68 \%$

Preparation of Methyl 4-(1-Methylethoxy)-3-Hydroxybutanoate (2i) [Table 2, Entry 9]

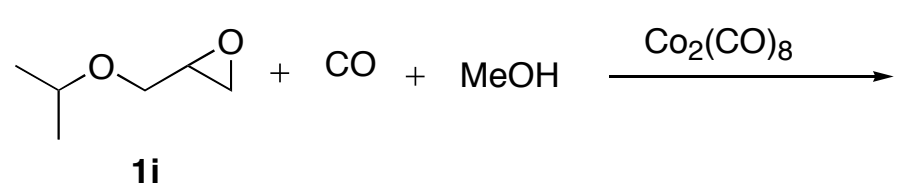

$\mathbf{1 i}$

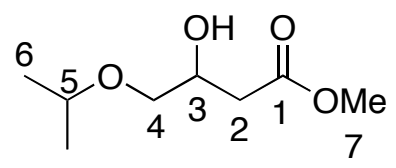

2i

Following General Procedure I, $\mathrm{Co}_{2}(\mathrm{CO})_{8}(84.5 \mathrm{mg}, 0.25 \mathrm{mmol}, 0.05$ equiv. $)$, freshly distilled methanol $(5.0 \mathrm{~mL})$ and $1 \mathbf{i}(632 \mu \mathrm{L}, 5.0 \mathrm{mmol})$ were stirred under a $\mathrm{CO}$ atmosphere $(1 \mathrm{~atm})$ at room temperature for $24 \mathrm{~h}$. The reaction mixture was diluted with diethyl ether $(50 \mathrm{~mL})$ and was stirred for $30 \mathrm{~min}$ in air, then passed through silica gel $(20 \mathrm{X} 100 \mathrm{~mm})$, with diethyl ether (10 $\mathrm{mL}$ ) as the eluent. Careful removal of solvent in vacuo followed by column chromatography 
$\left(\mathrm{SiO}_{2}, 20 \mathrm{~mm} \mathrm{X} 20 \mathrm{~cm}\right.$, hexane/EtOAc, hexane, 20/1, 3/1) to afforded $703 \mathrm{mg}(80 \%)$ of $\mathbf{2 i}$ as clear colorless oil.

Data for 2i:

bp $\quad 95^{\circ} \mathrm{C}$ at $0.5 \mathrm{mmHg}$

${ }^{1} \mathrm{H} \mathrm{NMR} \quad\left(500 \mathrm{MHz}, \mathrm{CDCl}_{3}\right)$

4.13 (m, $1 \mathrm{H}, \mathrm{HC}(3)), 3.68$ (s, $3 \mathrm{H}, \mathrm{HC}(7)), 3.57$ (hept, $J=12.2,6.1,1 \mathrm{H}, \mathrm{HC}(5))$, $3.43(\mathrm{dd}, J=9.3,4.5,1 \mathrm{H}, \mathrm{HC}(4)), 3.35(\mathrm{dd}, J=9.5,6.1,1 \mathrm{H}, \mathrm{HC}(4)), 2.97$ (d, $J=$ 4.6, $1 \mathrm{H}, \mathrm{OH}), 2.51(\mathrm{~d}, J=6.1,2 \mathrm{H}, \mathrm{HC}(2)), 1.12(\mathrm{~d}, J=6.1,6 \mathrm{H}, \mathrm{HC}(6))$,

${ }^{13} \mathrm{C} \mathrm{NMR} \quad\left(125 \mathrm{MHz}, \mathrm{CDCl}_{3}\right)$

$172.4(\mathrm{C}(1)), 72.1(\mathrm{C}(5)), 71.0(\mathrm{C}(4)), 67.3(\mathrm{C}(3)), 51.7(\mathrm{C}(7)), 38.1(\mathrm{C}(2)), 22.0$

$(\mathrm{C}(6)), 21.9(\mathrm{C}(6))$

IR (neat)

3450 (s), 2973 (s), 2930 (s), 2873 (s), 2630 (w), 1736 (s), 1438 (s), 1369 (s), 1336

(s), 1256 (s), 1163 (s), 1129 (s), 1075 (s), 1007 (m), 938 (m), 1599 (m), 884 (w), $824(w)$

$\underline{\mathrm{MS}} \quad(\mathrm{CI}, 70 \mathrm{eV})$

$177\left(\mathrm{M}^{+1}, 100\right), 159$ (55), 145 (62), 135 (74), 117 (75), 103 (80), 85 (69), 71 (37), $61(42)$

TLC $R f 0.22$ (hexane/EtOAc, 4/1) [silica gel, p-anisaldehyde stain]

$\underline{\text { Analysis }} \quad \mathrm{C}_{8} \mathrm{H}_{16} \mathrm{O}_{4}(176.21)$

Calculated: $\quad$ C, 54.53; $\quad$ H, $9.15 \%$

Found: $\quad$ C, 54.44; $\quad$ H, $9.52 \%$ 
Preparation of Methyl 4-[[(1,1-Dimethylethyl)dimethylsilyl]oxy]-3-Hydroxybutanoate (2j) [Table 2, Entry 10]

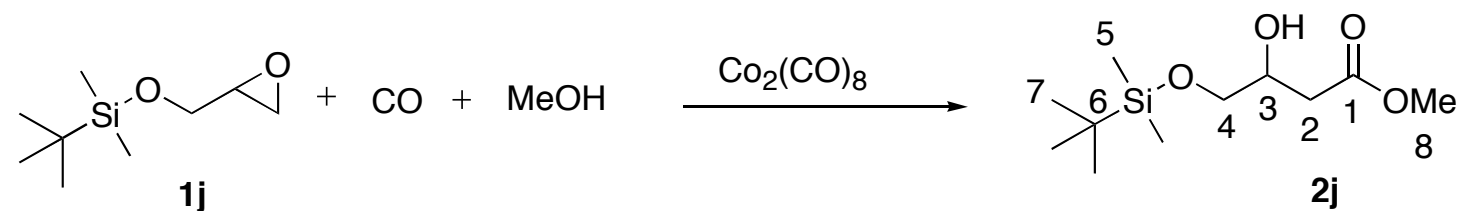

Following General Procedure I, $\mathrm{Co}_{2}(\mathrm{CO})_{8}(84.5 \mathrm{mg}, 0.25 \mathrm{mmol}, 0.05$ equiv. $)$, freshly distilled methanol $(5.0 \mathrm{~mL})$ and $\mathbf{1 j}(1000 \mu \mathrm{L}, 5.0 \mathrm{mmol})$ were stirred under a $\mathrm{CO}$ atmosphere $(1$ atm) at room temperature for $24 \mathrm{~h}$. The reaction mixture was diluted with diethyl ether (50 $\mathrm{mL})$ and was stirred for $30 \mathrm{~min}$ in air, then passed through silica gel (20 X $100 \mathrm{~mm})$, with diethyl ether $(10 \mathrm{~mL})$ as the eluent. Careful removal of solvent in vacuo followed by column chromatography $\left(\mathrm{SiO}_{2}, 20 \mathrm{~mm}\right.$ X $20 \mathrm{~cm}$, hexane/EtOAc, hexane, 20/1, 4/1) to afforded $272 \mathrm{mg}$ (22\%) of $\mathbf{2} \mathbf{j}$ as clear colorless oil.

Data for 2j:

bp $\quad 135^{\circ} \mathrm{C}$ at $0.5 \mathrm{mmHg}$

${ }^{1} \mathrm{H} \mathrm{NMR} \quad\left(500 \mathrm{MHz}, \mathrm{CDCl}_{3}\right)$

4.08 (m, 1 H, HC(3)), 3.71 (s, 3 H, HC(8)), 3.63 (dd, J=10.0, 4.8, 1 H, HC(4)), $3.56(\mathrm{dd}, J=10.0,5.8,1 \mathrm{H}, \mathrm{HC}(4)), 2.83(\mathrm{~d}, J=4.8,1 \mathrm{H}, \mathrm{OH}), 2.52(\mathrm{~m}, 2 \mathrm{H}$, $\mathrm{HC}(2)), 0.89$ (s, $9 \mathrm{H}, \mathrm{HC}(7)), 0.06$ (s, $6 \mathrm{H}, \mathrm{HC}(5))$

${ }^{13} \mathrm{C} \mathrm{NMR} \quad\left(125 \mathrm{MHz}, \mathrm{CDCl}_{3}\right)$

$172.5(\mathrm{C}(1)), 68.5(\mathrm{C}(3)), 66.1(\mathrm{C}(4)), 51.8(\mathrm{C}(8)), 37.7(\mathrm{C}(2)), 25.8(\mathrm{C}(7)), 18.3$ $(\mathrm{C}(6)),-5.45(\mathrm{C}(5)),-5.47(\mathrm{C}(5))$

$\underline{\text { IR }} \quad$ (neat)

3475 (s), 2950 (s), 2930 (s), 2858 (s), 2733 (w), 2713 (w), 1736 (s), 1463 (s), 1438 (s), 1362 (s), 1253 (s), 1169 (s), 1119 (s), 1072 (s), 1006 (m), 939 (m), 836 
(s), $777(\mathrm{~s}), 670(\mathrm{~m})$

MS

(CI, $70 \mathrm{eV})$

$249\left(\mathrm{M}^{+1}, 84\right), 233$ (82), 217 (100), 191 (90), 173 (88), 159 (90), 131 (75), 117

(91), 85 (67), 75 (66), 59 (75)

TLC $\quad R f 0.23$ (hexane/EtOAc, 4/1) [silica gel, p-anisaldehyde stain]

Analysis $\quad \mathrm{C}_{11} \mathrm{H}_{24} \mathrm{O}_{4} \mathrm{Si}(176.21)$

Calculated: $\quad$ C, $53.19 ; \quad$ H, $9.74 \%$

Found: $\quad$ C, $52.92 ; \quad H, 9.99 \%$

Preparation of Methyl 4-(N-Phthalimido)-3-Hydroxybutanoate (2k) [Table 2, Entry 11]
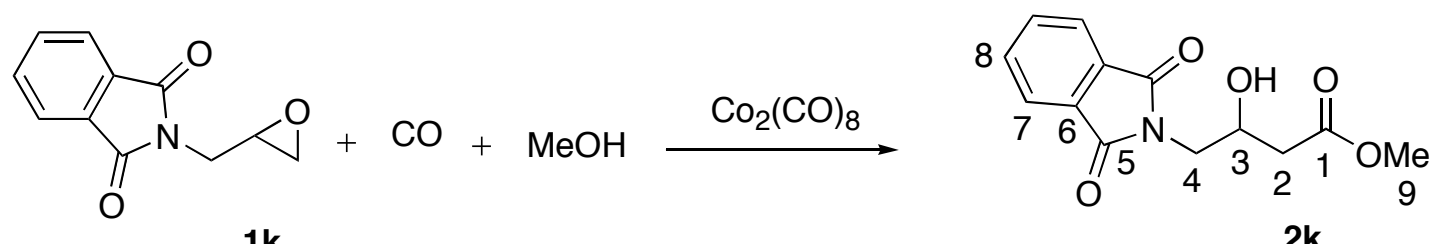

Following General Procedure I, $\mathrm{Co}_{2}(\mathrm{CO})_{8}(84.5 \mathrm{mg}, 0.25 \mathrm{mmol}, 0.05$ equiv. $)$, freshly distilled methanol $(5.0 \mathrm{~mL})$ and $1 \mathbf{k}(1.01 \mathrm{~g}, 5.0 \mathrm{mmol})$ were stirred under a $\mathrm{CO}$ atmosphere $(1 \mathrm{~atm})$ at room temperature for $24 \mathrm{~h}$. The reaction mixture was diluted with diethyl ether $(50 \mathrm{~mL})$ and was stirred for $30 \mathrm{~min}$ in air, then passed through silica gel $(20 \mathrm{X} 100 \mathrm{~mm})$, with diethyl ether $(10$ $\mathrm{mL)}$ as the eluent. Careful removal of solvent in vacuo followed by column chromatography $\left(\mathrm{SiO}_{2}, 20 \mathrm{~mm} \mathrm{X} 20 \mathrm{~cm}\right.$, hexane/EtOAc, hexane, 20/1, 1.5/1) to afforded $975 \mathrm{mg}(22 \%)$ of $\mathbf{2 k}$ as white solid.

Data for $2 \mathrm{k}$ :

$\underline{m p}$ $124-126^{\circ} \mathrm{C}$ 
${ }^{1} \mathrm{H} \mathrm{NMR} \quad\left(500 \mathrm{MHz}, \mathrm{CDCl}_{3}\right)$

$7.86(\mathrm{dd}, J=5.3,2.9,2 \mathrm{H}, \mathrm{HC}(8)), 7.73(\mathrm{dd}, J=5.4,3.0,2 \mathrm{H}, \mathrm{HC}(7)), 4.36(\mathrm{~m}, 1$ H, HC(3)), 3.89 (dd, $J=14.0,7.2,1 \mathrm{H}, \mathrm{HC}(4)), 3.77$ (dd, $J=13.8,4.6,1 \mathrm{H}$, $\mathrm{HC}(4)), 3.71(\mathrm{~s}, 3 \mathrm{H}, \mathrm{HC}(9)), 3.2(\mathrm{~d}, J=4.8,1 \mathrm{H}, \mathrm{OH}), 2.61(\mathrm{dd}, J=16.6,3.9,1$ H, $\mathrm{HC}(2)), 2.53(\mathrm{dd}, J=16.6,8.3,1 \mathrm{H}, \mathrm{HC}(2))$

${ }^{13} \mathrm{C} \mathrm{NMR} \quad\left(125 \mathrm{MHz}, \mathrm{CDCl}_{3}\right)$

$172.0(\mathrm{C}(1)), 168.4(\mathrm{C}(5)), 134.0(\mathrm{C}(6)), 131.7(\mathrm{C}(8)), 123.3(\mathrm{C}(7)), 66.4(\mathrm{C}(3))$, $51.8(\mathrm{C}(9)), 42.8(\mathrm{C}(4)), 38.8(\mathrm{C}(2))$

$\underline{\text { IR }} \quad$ (neat)

$3480(w), 2952(w), 1773(w), 1711(\mathrm{~s}), 1467(w), 1435(w), 1395(\mathrm{~m}), 1301(w)$, $1192(\mathrm{w}), 1172(\mathrm{w}), 1058(\mathrm{w}), 1023(\mathrm{w}), 884(\mathrm{w}), 725(\mathrm{~m})$

$\underline{\mathrm{MS}}$

(CI, $70 \mathrm{eV})$

$264\left(\mathrm{M}^{+1}, 93\right), 260$ (1), 247 (3), 246 (19), 245, (4), 232 (15), 214 (58), 186 (43), 162 (16), 161 (100), 160 (52), 133 (32), 132 (19), 117 (25), 105 (21), 104 (27), TLC Rf0.21 (hexane/EtOAc, 1/1) [silica gel, p-anisaldehyde stain] Analysis $\quad \mathrm{C}_{13} \mathrm{H}_{13} \mathrm{NO}_{5}(263.25)$
Calculated:
C, 59.31;
H, 4.98
$\mathrm{N}, 5.32 \%$

Found:

C, 59.12; H, 4.92

$\mathrm{N}, 5.35 \%$ 


\section{Preparation of Methyl 5,5-Diethoxy-3-Hydroxybutanoate (21) [Table 2, Entry 12]}

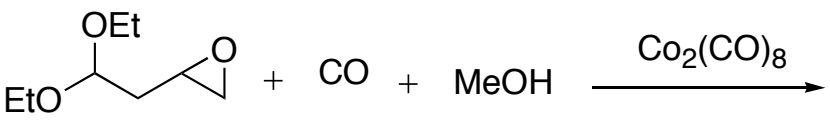

11

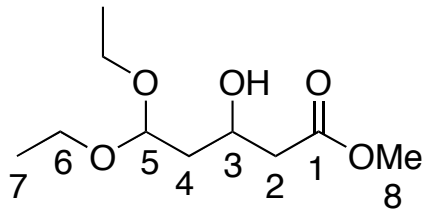

2I

Following General Procedure $\mathrm{I}, \mathrm{Co}_{2}(\mathrm{CO})_{8}(84.5 \mathrm{mg}, 0.25 \mathrm{mmol}, 0.05$ equiv.), freshly distilled methanol $(5.0 \mathrm{~mL})$ and $\mathbf{1 l}(800 \mu \mathrm{L}, 5.0 \mathrm{mmol})$ were stirred under a $\mathrm{CO}$ atmosphere $(1 \mathrm{~atm})$ at room temperature for $24 \mathrm{~h}$. The reaction mixture was diluted with diethyl ether $(50 \mathrm{~mL})$ and was stirred for $30 \mathrm{~min}$ in air, then passed through silica gel (20 X $100 \mathrm{~mm})$, with diethyl ether (10 $\mathrm{mL}$ ) as the eluent. Careful removal of solvent in vacuo followed by column chromatography $\left(\mathrm{SiO}_{2}, 20 \mathrm{~mm} \mathrm{X} 20 \mathrm{~cm}\right.$, hexane/EtOAc, hexane, 20/1, 4/1) to afforded $120 \mathrm{mg}(11 \%)$ of $2 \mathbf{l}$ as clear colorless oil.

Data for 2l:

bp $\quad 80{ }^{\circ} \mathrm{C}$ at $0.5 \mathrm{mmHg}$

${ }^{1} \mathrm{H} \mathrm{NMR} \quad\left(400 \mathrm{MHz}, \mathrm{CDCl}_{3}\right)$

$7.86(\mathrm{t}, J=5.4,10.9,1 \mathrm{H}, \mathrm{HC}(5)), 4.23(\mathrm{~m}, 1 \mathrm{H}, \mathrm{HC}(3)), 3.70$ (s, $3 \mathrm{H}, \mathrm{HC}(8))$, $3.66(\mathrm{~m}, 2 \mathrm{H}, \mathrm{HC}(6)), 3.52$ (m, $2 \mathrm{H}, \mathrm{HC}(6)), 3.52$ (m, $1 \mathrm{H}, \mathrm{OH}), 2.49$ (m, $2 \mathrm{H}$, $\mathrm{HC}(2)), 1.21(\mathrm{t}, J=14.1,7.0,3 \mathrm{H}, \mathrm{HC}(7)), 1.21(\mathrm{t}, J=13.9,6.9,3 \mathrm{H}, \mathrm{HC}(7))$

${ }^{13} \mathrm{C} \mathrm{NMR} \quad\left(125 \mathrm{MHz}, \mathrm{CDCl}_{3}\right)$

$172.6(\mathrm{C}(1)), 101.5(\mathrm{C}(5)), 65.1(\mathrm{C}(3)), 62.2(\mathrm{C}(6)), 61.9(\mathrm{C}(6)), 51.7(\mathrm{C}(8)), 41.4$ $(\mathrm{C}(4)), 39.9(\mathrm{C}(2)), 15.3(\mathrm{C}(7)), 15.3(\mathrm{C}(7))$

$\underline{\text { IR }} \quad$ (neat)

$3468(\mathrm{~m}), 2976(\mathrm{~m}), 2925(\mathrm{~m}), 1736(\mathrm{~s}), 1438(\mathrm{w}), 1374(\mathrm{w}), 1346(\mathrm{w}), 1266(\mathrm{w})$, $1168(\mathrm{~m}), 1126(\mathrm{~m}), 1060(\mathrm{~m}), 1008(\mathrm{w})$ 
$\underline{\mathrm{MS}}$

$(\mathrm{CI}, 70 \mathrm{eV})$

$243\left(\mathrm{M}^{+}+\mathrm{Na}, 3\right), 229$ (4), $219\left(\mathrm{M}^{-1}, 1\right), 211(1), 205$ (1), 191 (2), 185, (1), 177

(18), $163(1), 157$ (100), $145(16), 125$ (8)

TLC $\quad R_{f} 0.22$ (hexane/EtOAc, 4/1) [silica gel, p-anisaldehyde stain]

Analysis $\quad \mathrm{C}_{10} \mathrm{H}_{20} \mathrm{O}_{5}(220.26)$

Calculated: $\quad$ C, 54.53; $\quad$ H, $9.15 \%$

Found: $\quad$ C, $54.64 ; \quad$ H, $9.47 \%$

Preparation of Dimethyl 6-Ethyl-3-hydroxyhexanedioate (2m) [Table 2, Entry 13]<smiles>CCOC(=O)CCC1CO1</smiles>

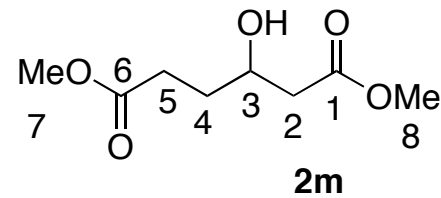

Following General Procedure I, $\mathrm{Co}_{2}(\mathrm{CO})_{8}(84.5 \mathrm{mg}, 0.25 \mathrm{mmol}, 0.05$ equiv. $)$, freshly distilled methanol $(5.0 \mathrm{~mL})$ and $1 \mathrm{~m}(720 \mu \mathrm{L}, 5.0 \mathrm{mmol})$ were stirred under a $\mathrm{CO}$ atmosphere $(1$ atm) at room temperature for $24 \mathrm{~h}$. The reaction mixture was diluted with diethyl ether (50 $\mathrm{mL})$ and was stirred for $30 \mathrm{~min}$ in air, then passed through silica gel (20 X $100 \mathrm{~mm})$, with diethyl ether $(10 \mathrm{~mL})$ as the eluent. Careful removal of solvent in vacuo followed by column chromatography $\left(\mathrm{SiO}_{2}, 20 \mathrm{~mm}\right.$ X $20 \mathrm{~cm}$, hexane/EtOAc, hexane, 20/1, 4/1) to afforded $185 \mathrm{mg}$ (18\%) of $\mathbf{2 m}$ as clear colorless oil. NMR spectral data matched that previously reported in the literature. $^{15}$

Data for $\mathbf{2 m}$ :

${ }^{1} \mathrm{H} \mathrm{NMR} \quad\left(500 \mathrm{MHz}, \mathrm{CDCl}_{3}\right)$

$4.04(\mathrm{~m}, 1 \mathrm{H}), 3.72(\mathrm{~s}, 3 \mathrm{H}), 3.68(\mathrm{~s}, 3 \mathrm{H}), 3.08$ (d, $1 \mathrm{H}), 1.75-1.88$ (m, $2 \mathrm{H}), 2.40-$

$2.55(\mathrm{~m}, 4 \mathrm{H}), 2.42(\mathrm{dd}, J=16.2,8.7,1 \mathrm{H}), 1.22(\mathrm{~d}, 3 \mathrm{H})$ 
${ }^{13} \mathrm{C} \mathrm{NMR} \quad\left(125 \mathrm{MHz}, \mathrm{CDCl}_{3}\right)$

$174.1,173.0,67.1,51.8,51.7,41.1,31.2,30.2$

IR (neat)

3530, 2950, 1740, $1674(\mathrm{w}), 1458(\mathrm{~m}), 1375(\mathrm{w}), 1283(\mathrm{~m}), 1243(\mathrm{~m}), 1169(\mathrm{~m})$,

$1119(\mathrm{~m}), 1041(\mathrm{w}), 983(\mathrm{w}), 855(\mathrm{w}), 788(\mathrm{w})$

Preparation of Dimethyl 2-Hydroxybutanedioate (2n) [Table 2, Entry 14]<smiles>COC(=O)C1CC1C(=O)O</smiles>

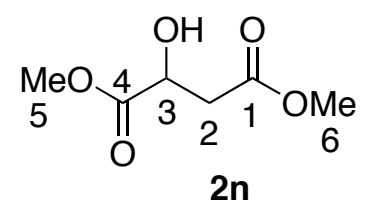

Following General Procedure I, $\mathrm{Co}_{2}(\mathrm{CO})_{8}(84.5 \mathrm{mg}, 0.25 \mathrm{mmol}, 0.05$ equiv. $)$, freshly distilled methanol $(5.0 \mathrm{~mL})$ and $\mathbf{1 n}(440 \mu \mathrm{L}, 5.0 \mathrm{mmol})$ were stirred under a CO atmosphere (1 atm) at room temperature for $24 \mathrm{~h}$. The reaction mixture was diluted with diethyl ether (50 $\mathrm{mL})$ and was stirred for $30 \mathrm{~min}$ in air, then passed through silica gel (20 X $100 \mathrm{~mm})$, with diethyl ether $(10 \mathrm{~mL})$ as the eluent. Careful removal of solvent in vacuo followed by column chromatography $\left(\mathrm{SiO}_{2}, 20 \mathrm{~mm}\right.$ X $20 \mathrm{~cm}$, hexane/EtOAc, hexane, 20/1, 4/1) to afforded $283 \mathrm{mg}$ (35\%) of $\mathbf{2 n}$ as clear colorless oil. NMR spectral data matched that previously reported in the literature. $^{16}$

Data for 2n:

${ }^{1} \mathrm{H} \mathrm{NMR} \quad\left(500 \mathrm{MHz}, \mathrm{CDCl}_{3}\right)$

4.49 (m, 1 H), 3.80 (s, 3 H), 3.70 (s, 3 H), 3.27 (d, 1 H), 2.86 (dd, $J=16.4,4.2,1$

$\mathrm{H}), 2.78(\mathrm{dd}, J=16.6,6.1,1 \mathrm{H})$

${ }^{13} \mathrm{C} \mathrm{NMR} \quad\left(125 \mathrm{MHz}, \mathrm{CDCl}_{3}\right)$

$173.7,170.9,67.2,52.8,52.0,38.3$ 
$\underline{\text { IR }}$ (neat)

$3458(\mathrm{~m}), 3008(\mathrm{w}), 2958(\mathrm{w}), 1731(\mathrm{~s}), 1640(\mathrm{w}), 1440(\mathrm{~m}), 1370(\mathrm{w}), 1278(\mathrm{~m})$, $1223(\mathrm{~m}), 1173(\mathrm{~m}), 1110(\mathrm{~m}), 1045(\mathrm{w}), 992(\mathrm{w}), 849(\mathrm{w}), 786(\mathrm{w})$

\section{Preparation of Methyl 3-Hydroxy-6-oxo-heptanoate (20) [Table 2, Entry 15]}

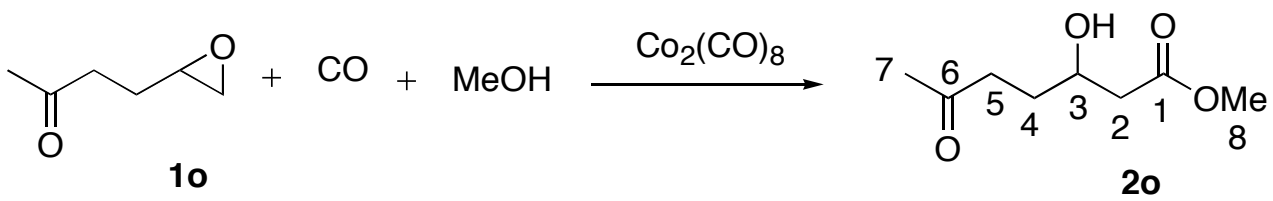

Following General Procedure I, $\mathrm{Co}_{2}(\mathrm{CO})_{8}(84.5 \mathrm{mg}, 0.25 \mathrm{mmol}, 0.05$ equiv. $)$, freshly distilled methanol $(5.0 \mathrm{~mL})$ and $10(570 \mu \mathrm{L}, 5.0 \mathrm{mmol})$ were stirred under a $\mathrm{CO}$ atmosphere $(1$ atm) at room temperature for $24 \mathrm{~h}$. The reaction mixture was diluted with diethyl ether (50 $\mathrm{mL})$ and was stirred for $30 \mathrm{~min}$ in air, then passed through silica gel (20 X $100 \mathrm{~mm})$, with diethyl ether $(10 \mathrm{~mL})$ as the eluent. Careful removal of solvent in vacuo followed by column chromatography $\left(\mathrm{SiO}_{2}, 20 \mathrm{~mm}\right.$ X $20 \mathrm{~cm}$, hexane/EtOAc, hexane, 20/1, 4/1) to afforded $148 \mathrm{mg}$ (17\%) of $\mathbf{2 0}$ as clear colorless oil.

\section{Data for 2o:}

bp $\quad 170{ }^{\circ} \mathrm{C}$ at $0.5 \mathrm{mmHg}$

${ }^{1} \mathrm{H} \mathrm{NMR} \quad\left(400 \mathrm{MHz}, \mathrm{CDCl}_{3}\right)$

3.98 (m, $1 \mathrm{H}, \mathrm{HC}(3)), 3.71(\mathrm{~s}, 3 \mathrm{H}, \mathrm{HC}(8)), 3.12$ (d, $J=3.41,1 \mathrm{H}, \mathrm{OH}), 2.64(\mathrm{~m}$, $2 \mathrm{H}, \mathrm{HC}(5)), 2.52(\mathrm{~d}, J=16.6,3.4,1 \mathrm{H}, \mathrm{HC}(2)), 2.44(\mathrm{~d}, J=16.6,8.5,1 \mathrm{H}$, $\mathrm{HC}(2)), 2.17$ (s, $3 \mathrm{H}, \mathrm{HC}(7)), 1.76(\mathrm{~m}, 1 \mathrm{H}, \mathrm{HC}(4)), 1.70$ (m, $1 \mathrm{H}, \mathrm{HC}(4))$

${ }^{13} \mathrm{C} \mathrm{NMR} \quad\left(125 \mathrm{MHz}, \mathrm{CDCl}_{3}\right)$

$208.9(\mathrm{C}(6)), 173.2(\mathrm{C}(1)), 67.2(\mathrm{C}(3)), 51.8(\mathrm{C}(8)), 41.2(\mathrm{C}(2)), 39.6(\mathrm{C}(5)), 30.0$ (C(4)), $29.9(\mathrm{C}(7))$ 
$\underline{\text { IR }}$ (neat)

3461 (m), 2953 (m), 1736 (s), 1711 (s), 1437 (s), 1363 (s), 1283 (s), 1259 (s), 1197 (s), 1170 (s), 1095 (m), 1012 (m), 934 (w), 885 (w)

$\underline{\mathrm{MS}}$

$(\mathrm{ES}, 70 \mathrm{eV})$

$197\left(\mathrm{M}^{+}+\mathrm{Na}, 42\right), 174$ (5), 158 (13), 157 (100), 126 (1), 125 (10), 115 (13)

TLC $R f 0.25$ (hexane/EtOAc, 1/1) [silica gel, p-anisaldehyde stain]

Analysis $\quad \mathrm{C}_{8} \mathrm{H}_{14} \mathrm{O}_{4}(174.19)$

Calculated: $\quad$ C, 55.16; $\quad \mathrm{H}, 8.10 \%$

Found: $\quad$ C, 54.93; H, $8.00 \%$

\section{Preparation of trans Ethyl 2-Hydroxycyclohexanoate (2p) [Table 2, Entry 16]}

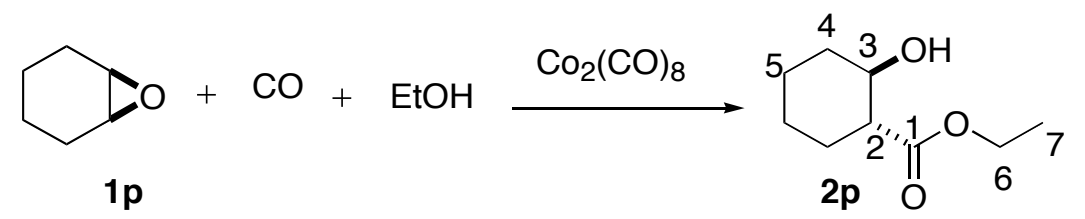

Following General Procedure I, $\mathrm{Co}_{2}(\mathrm{CO})_{8}(84.5 \mathrm{mg}, 0.25 \mathrm{mmol}, 0.05$ equiv. $)$, freshly distilled ethanol $(5.0 \mathrm{~mL})$ and $1 \mathbf{p}(506 \mu \mathrm{L}, 5.0 \mathrm{mmol})$ were stirred under a $\mathrm{CO}$ atmosphere $(1$ atm) at room temperature for $24 \mathrm{~h}$. The reaction mixture was diluted with diethyl ether (50 $\mathrm{mL})$ and was stirred for $30 \mathrm{~min}$., then passed through silica gel (20 X $100 \mathrm{~mm})$, with diethyl ether (10 $\mathrm{mL}$ ) as the eluent. Careful removal of solvent in vacuo followed by column chromatography $\left(\mathrm{SiO}_{2}, 20 \mathrm{~mm} \mathrm{X} 20 \mathrm{~cm}\right.$, hexane/EtOAc, hexane, 20/1, 4/1) to $175 \mathrm{mg}(20 \%)$ of $\mathbf{2 p}$ as clear colorless oil. NMR spectral data matched those previously reported in the literature. ${ }^{17}$

Data for 2p:

$\underline{{ }^{1} \mathrm{H} \mathrm{NMR}} \quad\left(500 \mathrm{MHz}, \mathrm{CDCl}_{3}\right)$

$4.16(\mathrm{q}, J=7.1,2 \mathrm{H}), 3.75(\mathrm{~m}, 1 \mathrm{H}), 2.82(\mathrm{~d}, J=3.4,1 \mathrm{OH}), 2.23(\mathrm{ddd}, J=9.2$, 
9.1, 3.2, 1 H), 2.01-2.06 (br m, 2 H), 1.75-1.78 (br m, 1 H), 1.69-1.73 (br m, 1 H), $1.27(\mathrm{t}, J=7.0,3 \mathrm{H}), 1.09-1.38($ br m, $4 \mathrm{H})$

${ }^{13} \mathrm{C} \mathrm{NMR} \quad\left(125 \mathrm{MHz}, \mathrm{CDCl}_{3}\right)$

$175.2,70.9,60.6,51.3,33.6,28.0,25.1,24.3,14.2$

$\underline{\mathrm{IR}}$

(neat)

3431 (s), 2980 (w), 2936 (s), 2860 (m), 1718 (s), 1450 (m), 1376 (m), 1323 (m),

$1251(\mathrm{~m}), 1229(\mathrm{~m}), 1180(\mathrm{~s}), 1067$ (s), 1034 (s), 987 (s), 952 (s), 860 (s)

\section{Preparation of $(S)$-Propylene Oxide ((S)-1a) [Scheme 2]}

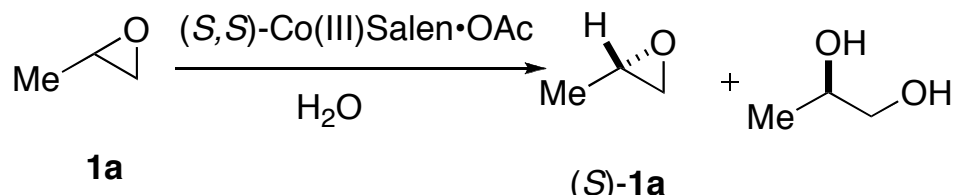

$(S)-1 a$

Following Jacobsen Procedure, ${ }^{18}(S$, )-Cobalt salen complex $(242 \mathrm{mg}, 400 \mu \mathrm{mol}, 0.002$ equiv.), in $5.0 \mathrm{~mL}$ toluene (from SDS) was treated with $240 \mu \mathrm{L} \mathrm{AcOH}$. The crude catalyst obtained after concentration was dissolved in propylene oxide (14.0 mL, $11.6 \mathrm{~g}, 200 \mathrm{mmol})$, and $\mathrm{H}_{2} \mathrm{O}\left(1.98 \mathrm{~mL}, 110 \mathrm{mmol}, 0.55\right.$ equiv) at $0{ }^{\circ} \mathrm{C}$ was added drop wise. After $14 \mathrm{~h}$ at $\mathrm{rt}$, propylene oxide (5.14 g, $44 \%$ ) was isolated by direct distillation at $34{ }^{\circ} \mathrm{C}$. The ${ }^{1} \mathrm{H}$ NMR spectrum matched that of the racemic epoxide.

Opt. Rot.: $\quad[\alpha]_{\mathrm{D}}^{23}-11.58$ (neat); lit $^{19}[\alpha]_{\mathrm{D}}^{23}-11.6$ (neat) 


\section{Determination of Enantiopurity of Propylene Oxide ((S)-1a)) [Scheme 2]}

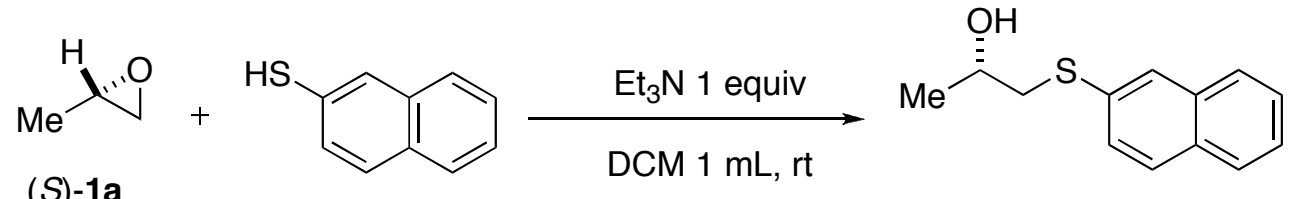
$(S)-1 a$

Oven-dried, 10-mL, round-bottomed flask was charged with 2-napthalenthiol (160 mg, $1.0 \mathrm{mmol}$ ) followed by $1 \mathrm{~mL}$ of dichloromethane and triethylamine $(134 \mu \mathrm{L}, 1.0 \mathrm{mmol}, 1.0$ equiv) and was stirred for $30 \mathrm{~s}$ whereupon the colorless mixture turned pale yellow. At this point (S)-propylene oxide $(70 \mu \mathrm{L}, 1.0 \mathrm{mmol})$ was added drop wise and the mixture was stirred for $6 \mathrm{~h}$ (monitored by TLC (silica gel plate, hexane/EtOAc 4/1). The reaction mixture was subjected to column chromatography $\left(\mathrm{SiO}_{2}, 20 \mathrm{~mm} \mathrm{X} 20 \mathrm{~cm}\right.$, hexane/EtOAc, hexane, 20/1, 4/1) to afford 110 $\operatorname{mg}(51 \%)$ of 1-(2-napthalenthio)-2-propanol. ${ }^{20}$

Data for 1-(2-napthalenthio)-2-propanol:

${ }^{1} \mathrm{H} \mathrm{NMR} \quad\left(500 \mathrm{MHz}, \mathrm{CDCl}_{3}\right)$

$7.83(\mathrm{~s}, 1 \mathrm{H}), 7.78(\mathrm{~m}, 3 \mathrm{H}), 7.47(\mathrm{~m}, 3 \mathrm{H}), 3.91(\mathrm{~m}, 1 \mathrm{H}), 3.20$ (dd, $J=13.6,3.9$, $1 \mathrm{H}), 2.95(\mathrm{dd}, J=13.7,8.4,1 \mathrm{H}), 2.60(\mathrm{~s}, 1 \mathrm{H}), 6.34(\mathrm{~d}, J=6.3,3 \mathrm{H})$

${ }^{13} \mathrm{C} \mathrm{NMR} \quad\left(125 \mathrm{MHz}, \mathrm{CDCl}_{3}\right)$

$133.1,132.5,131.9,128.5,128.1,127.7,127.6,127.0,126.6,125.9,65.6,43.4$, 21.9

$\underline{\mathrm{IR}}$ (neat)

3346 (s), 3043 (m), 2968 (s), 2909 (s), 1586 (m), 1371 (m), 1129 (m), 1074 (w), $1033(\mathrm{w}), 940(\mathrm{w}), 844(\mathrm{w}), 814(\mathrm{~m}), 737(\mathrm{~m})$

SFC: $\quad t_{\mathrm{R}} 6.30 \mathrm{~min}(98.4 \%) ; t_{\mathrm{R}} 6.95 \mathrm{~min}(1.6 \%)\left(\right.$ Chiralpak OD, $125 \mathrm{psi}, 40{ }^{\circ} \mathrm{C}, 10 \%$ $\mathrm{MeOH}$ in $\mathrm{CO}_{2}, 3.0 \mathrm{~mL} / \mathrm{min}, 220 \mathrm{~nm}$ ) 
Analysis $\quad \mathrm{C}_{13} \mathrm{H}_{14} \mathrm{OS}(218.31)$
Calculated:
C, 71.52;
$\mathrm{H}, 6.46 \%$
Found:
C, 71.50;
$\mathrm{H}, 6.50 \%$

Preparation of $(S)$ Methyl 3-Hydroxybutanoate (2a) [Scheme 2]

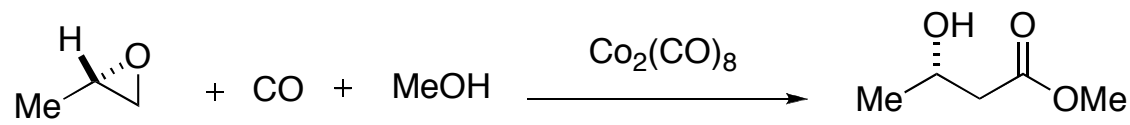

$(S)-1 \mathrm{a}$

$(S)-2 a$

Following General Procedure I, $\mathrm{Co}_{2}(\mathrm{CO})_{8}(84.5 \mathrm{mg}, 0.25 \mathrm{mmol}, 0.05$ equiv. $)$, freshly distilled methanol $(5.0 \mathrm{~mL})$ and $(S)-1 \mathbf{a}(350 \mu \mathrm{L}, 5.0 \mathrm{mmol})$ were stirred under a CO atmosphere (1 atm) at room temperature for $24 \mathrm{~h}$. The reaction mixture was diluted with diethyl ether (50 $\mathrm{mL}$ ) and was stirred for $30 \mathrm{~min}$, then passed through silica gel (20 X $100 \mathrm{~mm})$, with diethyl ether $(10 \mathrm{~mL})$ as the eluent. Careful removal of solvent under reduced pressure afforded $520 \mathrm{mg}$ $(88 \%)$ of $(S)-\mathbf{2 a}$ as clear, colorless oil. The ${ }^{1} \mathrm{H}$ NMR spectrum of this product matched that of the racemic material.

Opt. Rot.: $\quad[\alpha]_{\mathrm{D}}^{23}+25.74\left(\mathrm{c}=0.3, \mathrm{CHCl}_{3}\right) ; \mathrm{lit}^{21}[\alpha]_{\mathrm{D}}^{23}+24.2\left(\mathrm{c}=0.82, \mathrm{CHCl}_{3}\right)$

GC: $\quad t_{\mathrm{R}} 4.99 \min (>99.9 \%) ; t_{\mathrm{R}} 5.37 \mathrm{~min}(<0.1 \%)\left(\mathrm{G}-\mathrm{TA}, 80{ }^{\circ} \mathrm{C}\right.$, isothermal, $\mathrm{H}_{2}$ head pressure of $15 \mathrm{psi}$ )

\section{References}

(1) (a) Gais, H-J.; Jurgen, M.; Jadhav, V. J. Org. Chem. 2001, 66, 3384-3396. (b) Majetich, G.; Hicks, R. Synlett. 1996, 7, 649-651.

(2) Mordini, A.; Peruzzi, D.; Russo, F.; Valacchi, M.; Reginato, G.; Brandi, A. Tetrahedron 2005, 61, 3349-3360.

(3) Yohei, K.; Junji, I.; Kobayashi, Y. Tetrahedron Lett. 2002, 43, 2725-2729. 
(4) Williamson, R. M.; Pietersma, A. L.; Jameson, G. B.; Parker, E. J. Bioorg. Med. Chem. Lett. 2005, 15, 2339-2342.

(5) Liu, Z-Y.; Ji, J-X.; Li, B-G. J. Chem. Soc., Perkin Trans. 2000, 1, 3519-3521.

(6) Hansen, K. B.; Rabbat, P.; Springfield, S. A.; Devine, P. N.; Grabowski, E. J. J.; Reider, P. J Tetrahedron Lett. 2001, 42, 8743-8745.

(7) Payne, G. B. Tetrahedron Lett. 1962, 18, 763-765.

(8) (a) Ema, T.; Sugiyama, Y.; Fukumoto, M.; Moriya, H.; Cui, J.-N.; Sakai, M. J. Org. Chem. 1998, 63, 4996-5000. (b) Utaka, M.; Watabu, H.; Higashi, H.; Sakai, T.; Tsuboi.; Torii, S. J. Org. Chem. 1999, 55, 3917-3921. (c) Burk, M. J.; Harper, P. G. T.; Kalberg, C. S. J. Am. Chem. Soc. 1995, 117, 4423-4424. (d) Hinterding, K.; Jacobsen, E. N. J. Org. Chem. 1999, 64, 2164-2165.

(9) Utaka, M.; Watabu, H.; Higashi, H.; Sakai, T.; Tsuboi.; Torii, S. J. Org. Chem. 1999, $55,3917-3921$.

(10) (a) Hirama, M.; Uei, M. J. Am. Chem. Soc. 1982, 104, 4251-4253. (b) Hayakawa, K.; Nagatsugi, F.; Kanematsu, K. J. Org. Chem. 1988, 53, 860-863.

(11) Denmark, S. E.; Winter, S. B. D.; Su, X.; Wong, K.-T. J. Am. Chem. Soc. 1996, 118, 7404-7405.

(12) Denmark, S. E.; Winter, S. B. D.; Su, X.; Wong, K.-T. J. Am. Chem. Soc. 1996, 118, 7404-7405.

(13) (a) Elenkov, M.; Tang, M.; Hauer, L.; Bernhar, J.; Dick, B. Org. Lett. 2006, 8, 42274229. (b) Burk, M. J.; Harper, P. G. T.; Kalberg, C. S. J. Am. Chem. Soc. 1995, 117, 4423-4424. (c) Bradshaw, C. W.; Fu, H.; Shen, G. J.; Wong, C. H. J. Org. Chem. 1992, 57, 1526-1532. 
(14) (a) Kloetzing, R. J.; Thaler, T.; Knochel, P. Org. Lett. 2006, 6, 1125-1128. (b) Abood, N. A. Synth. Commun. 1993, 23, 811-815. (c) Washio, T.; Nakamura, S.; Anada, M.; Hashimoto, S. Heterocycles, 2005, 66, 567-568. (c) Syn. Commun., 1993, 23, 6, 811-815. (d) Hoffmann, T.; Seebach, D. Liebigs Ann. 1996, 1277-1282. (e) Hinterding, K.; Jacobsen, E. N. J. Org. Chem. 1999, 64, 2164-2165.

(15) Juskiewicz, G.; Asztemborska, M.; Jurczak, J. Synth. Commun. 2002, 32, $2605-$ 2611.

(16) (a) Larsen, C. H.; Ridgway, B. H.; Shaw, J. T.; Woerpel, K. A. J. Am. Chem. Soc. 1999, 121, 12208-12209. (b) MacNiel, P. A.; Roberts, N. K.; Bosnich, B. J. Am. Chem. Soc. 1981, 103, 2273-2280.

(17) Frater, G. Helv. Chim. Acta. 1980, 63, 1383-1390.

(18) Schaus, S. E.; Brandes, B. D.; Larrow, F. J.; Tokunaga, M.; Hansen, K. B.; Gould, A. E.; Furrow, M. E.; Jacobsen, E. N. J. Am. Chem. Soc. 2002, 124, 1307-1315.

(19) (a) Ellis, M. K.; Golding, B. T. Org. Synth., Coll. Vol. VII 1990, 356-359. (b) Shaus, S. E. Brandes, B. D.; Larrow, J. F.; Tokunga, M.; Hansen, K. B.; Gould, A. E.; Furrow, M. E.; Jacobsen, E. N. J. Am. Chem. Soc. 2002, 124, 1307-1315.

(20) Singh, S.; Kumar, S.; Chimni, S. S. Tetrahedron: Asymmetry 2001, 12, 2457-2462.

(21) Salvi, N. A.; Chattopadhyay, S. Tetrahedron: Asymmetry 2004, 15, 3397-3400. 


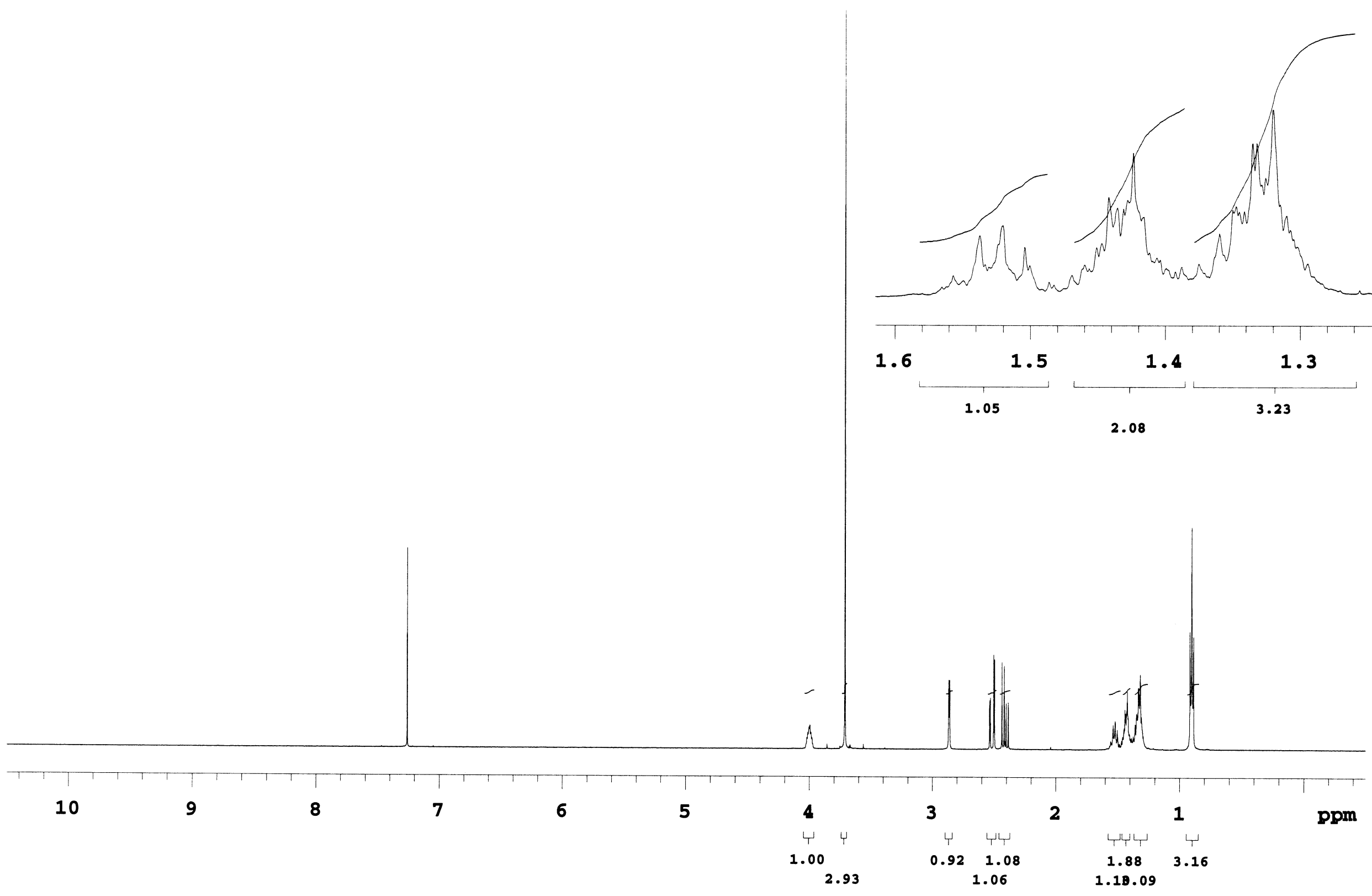



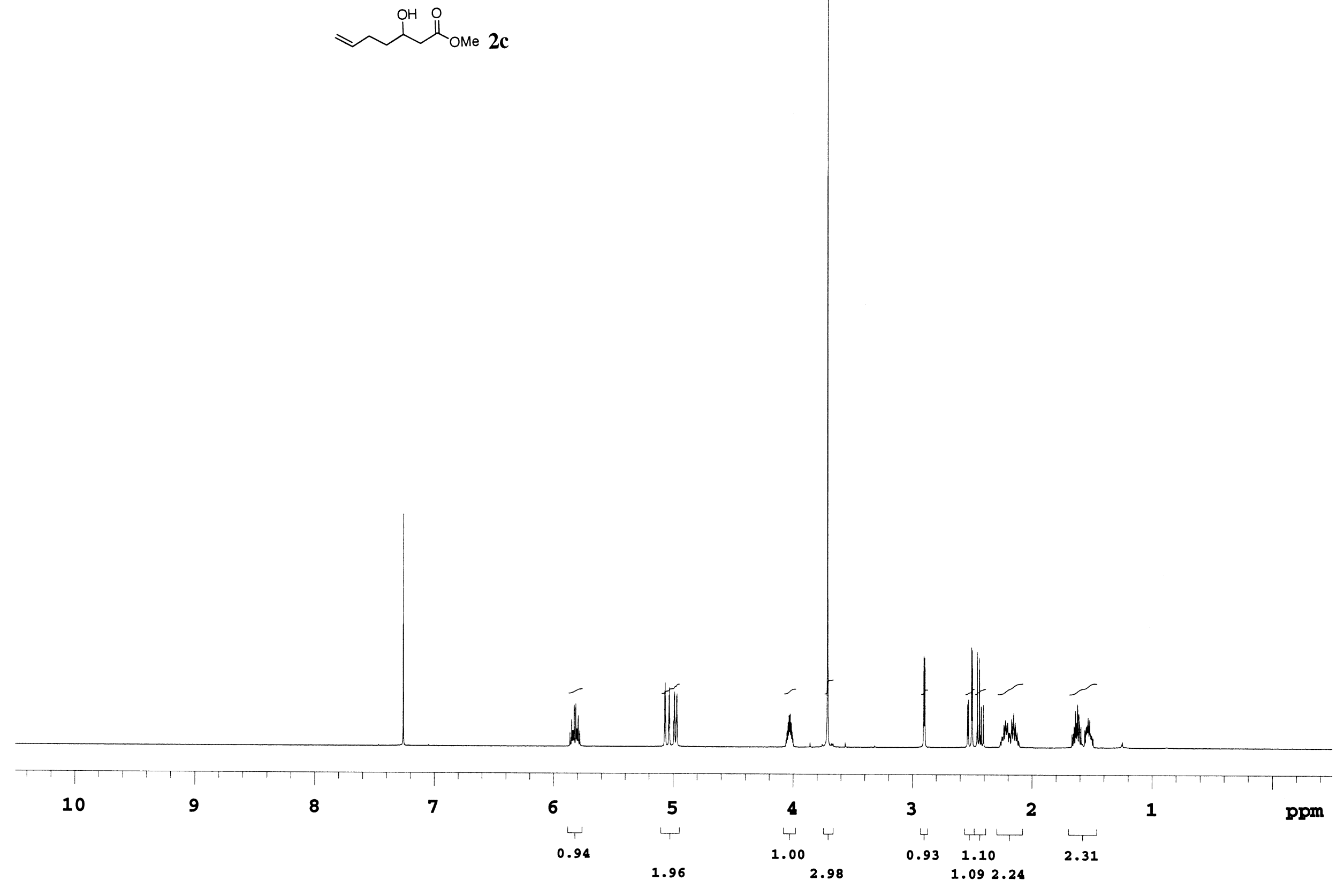

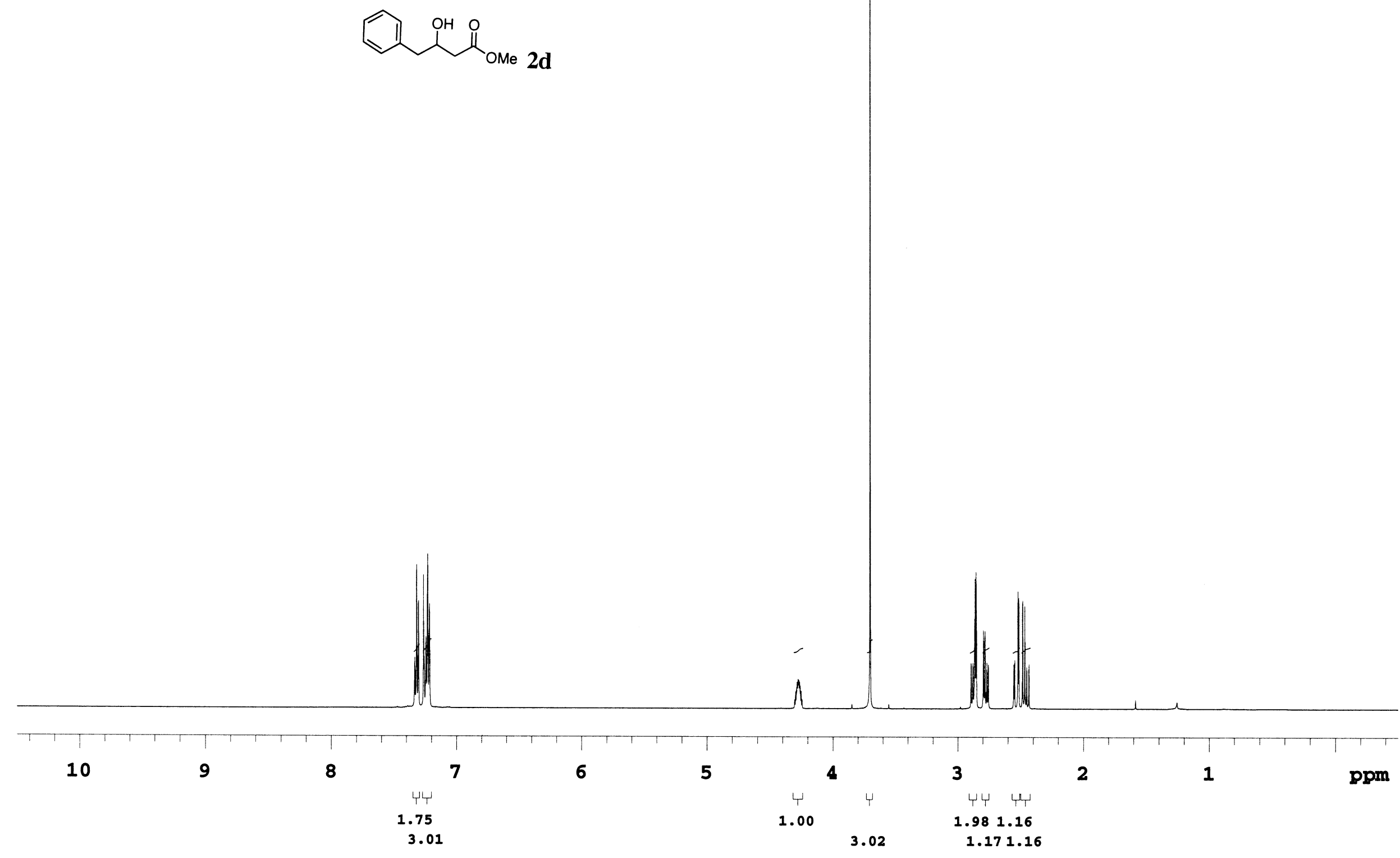


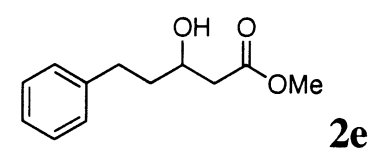

$2 \mathbf{e}$

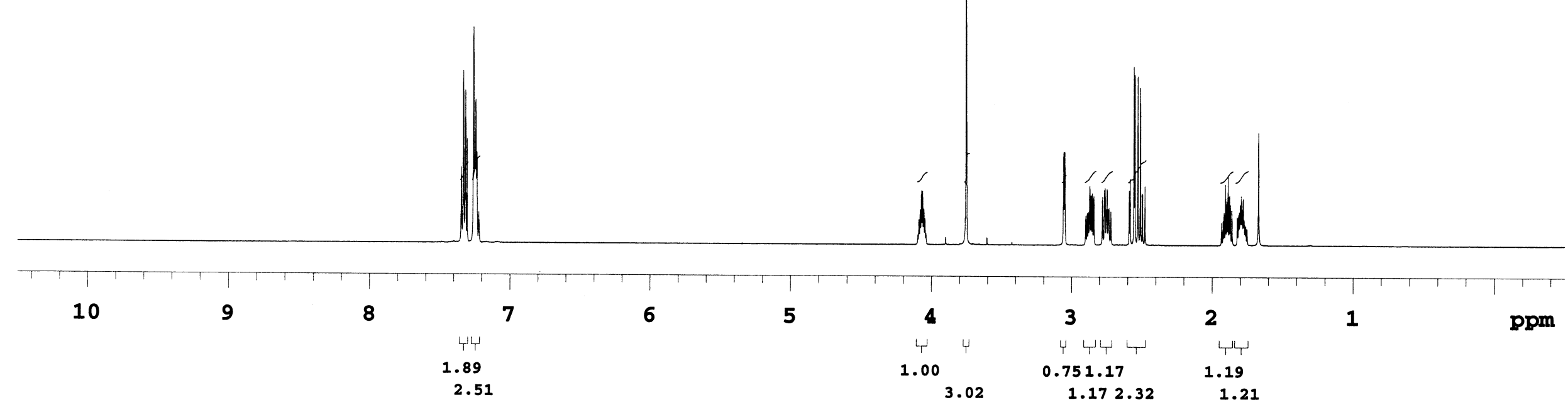




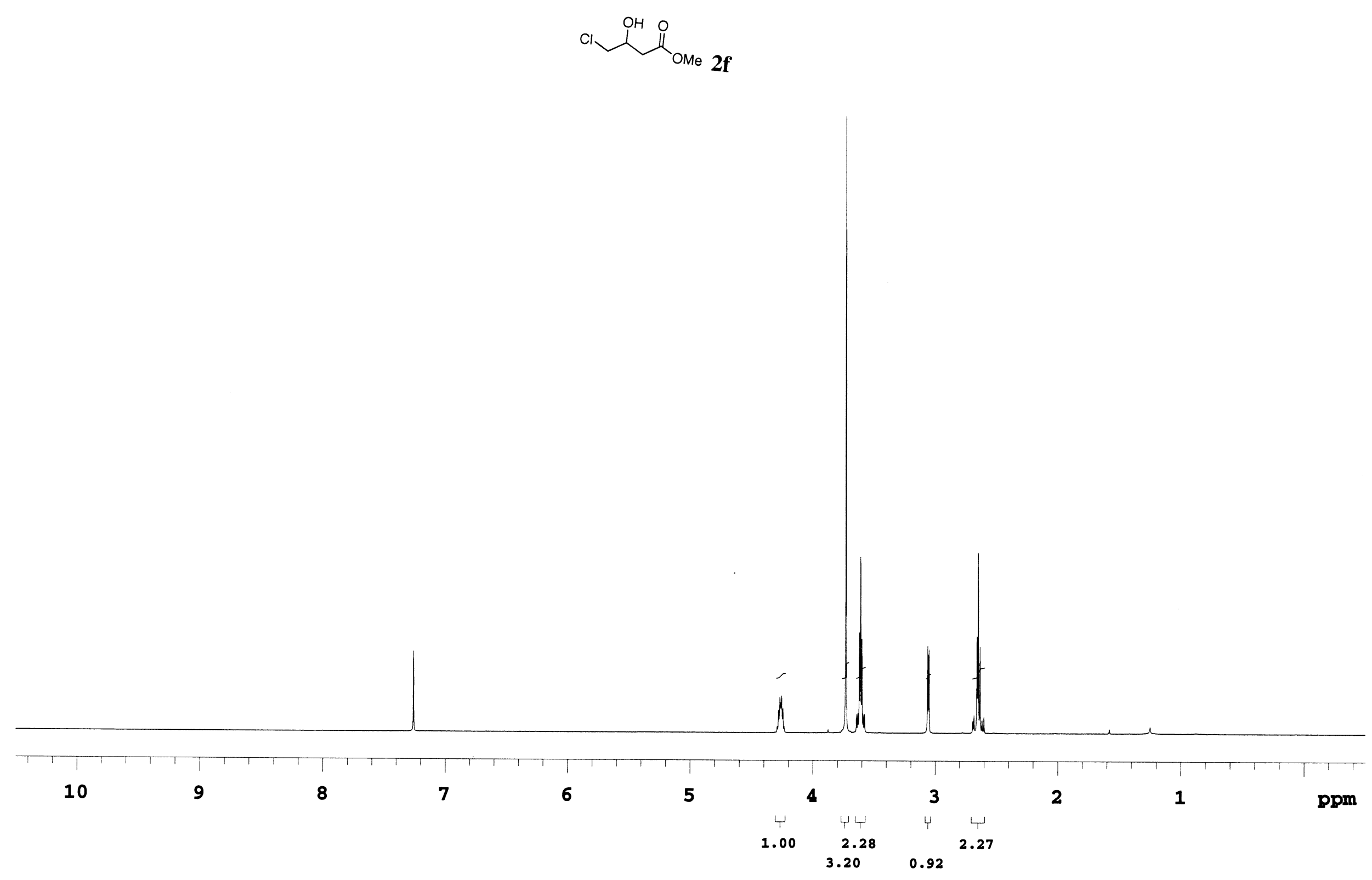




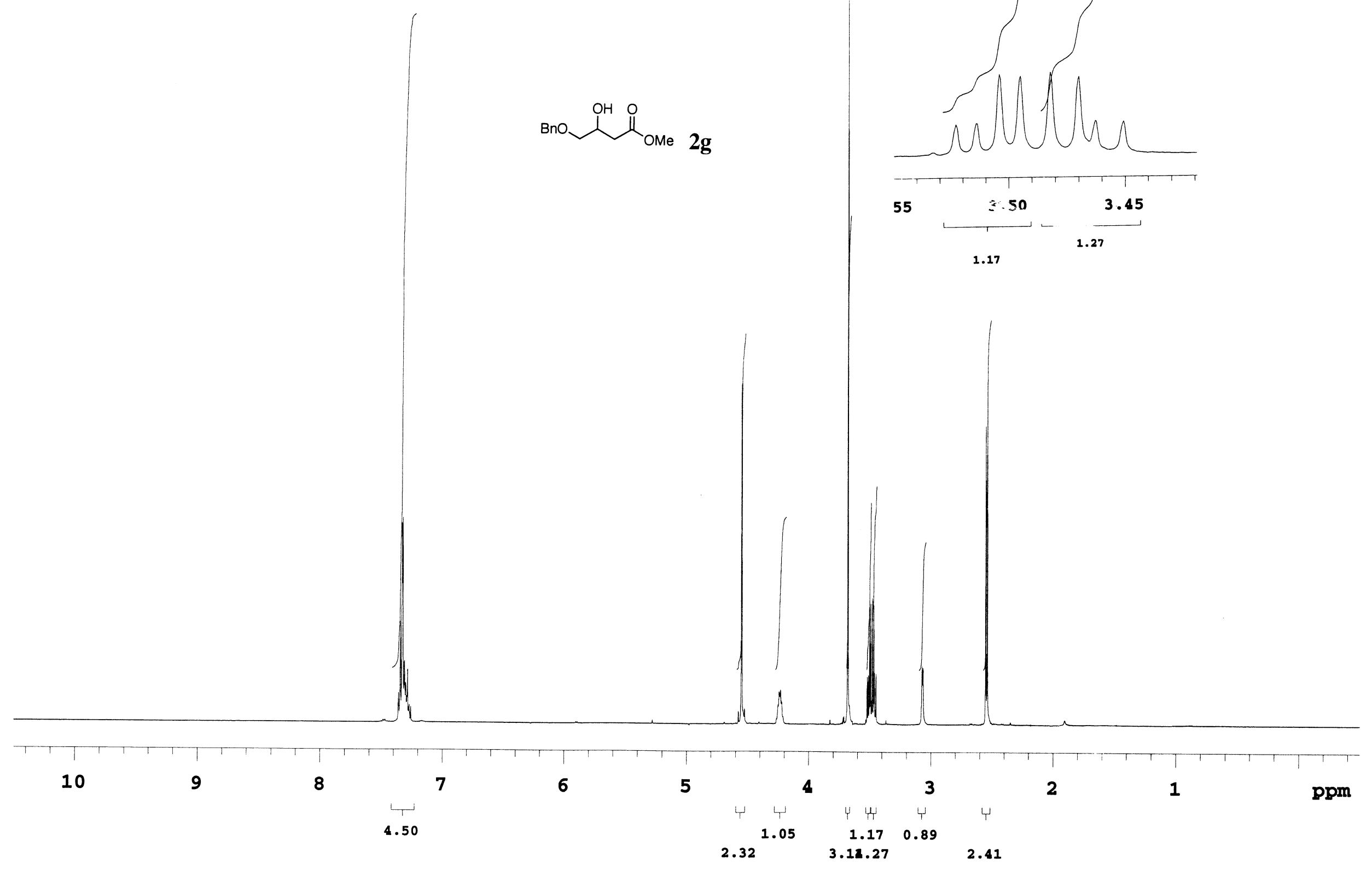




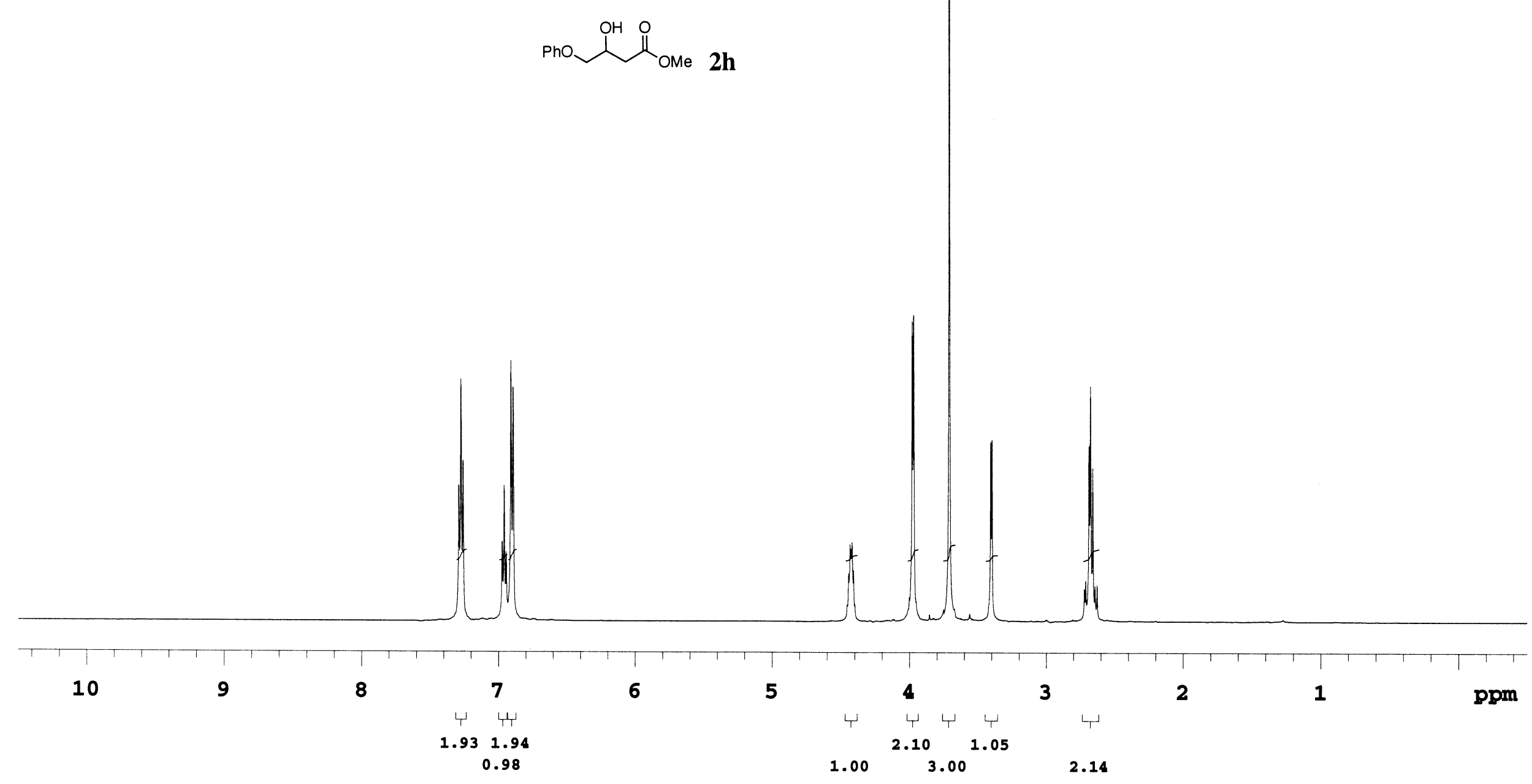


시을 $2 \mathbf{i}$

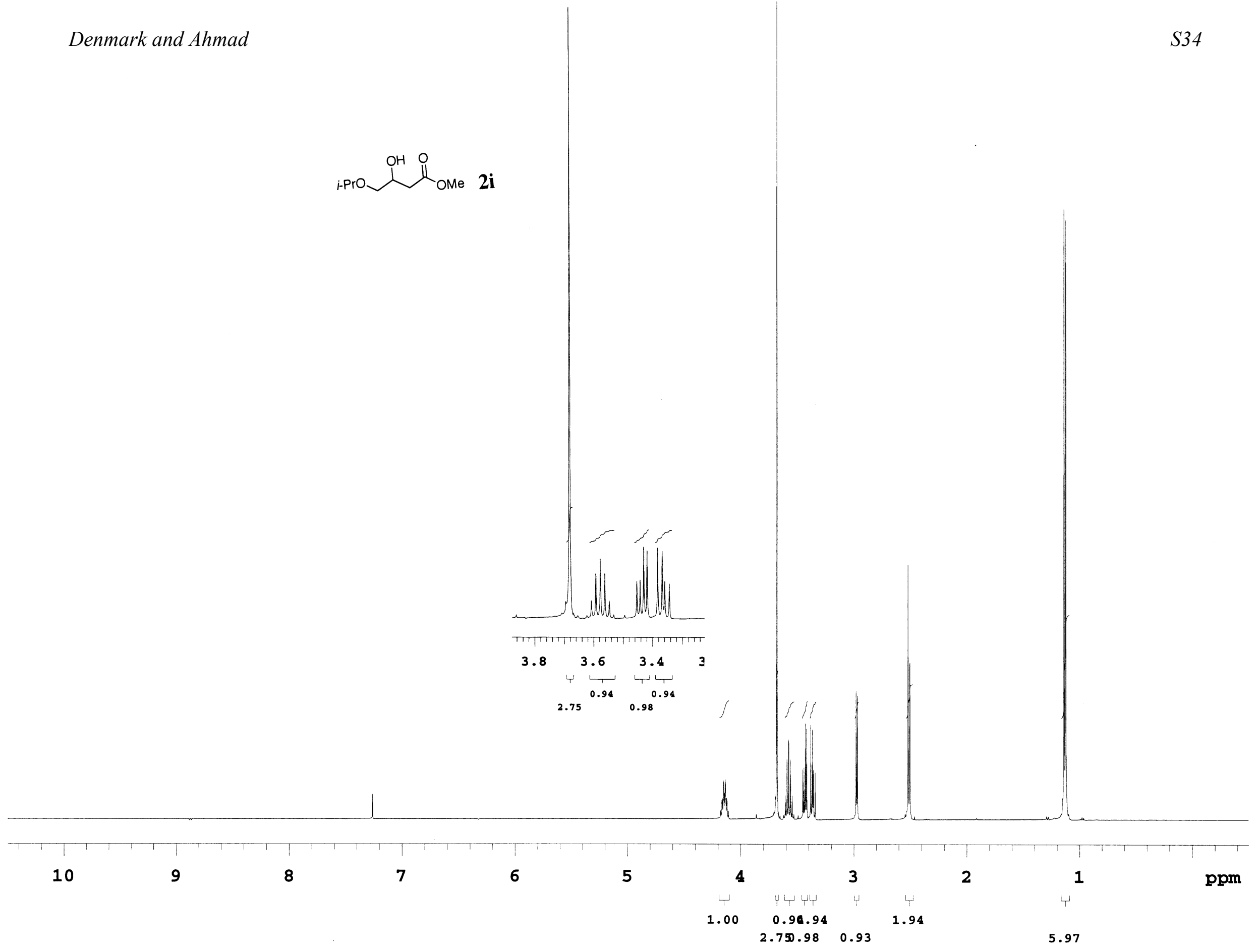



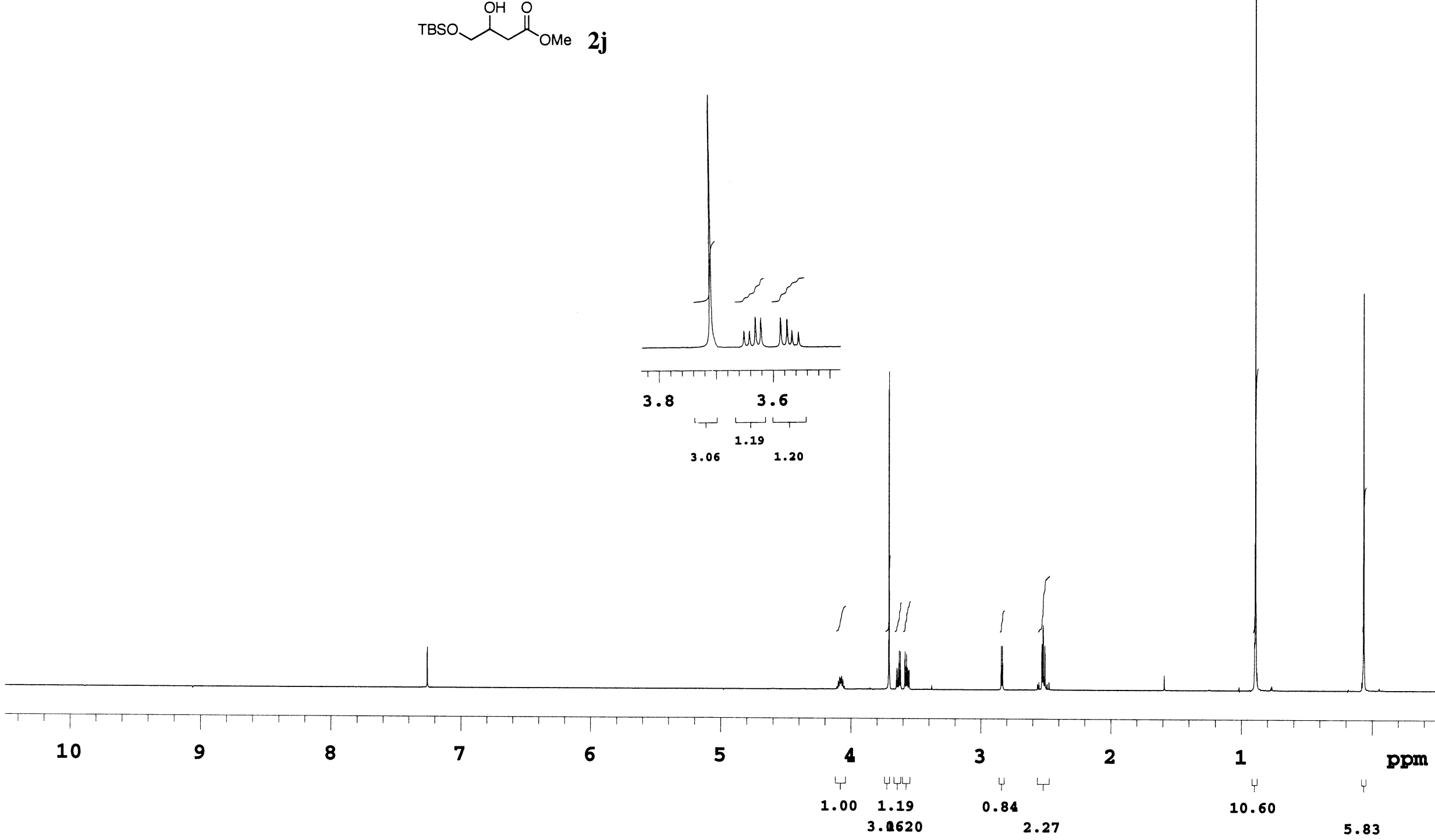


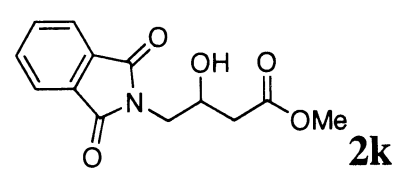

$2 \mathbf{k}$ 
$\overbrace{O M e} 21$

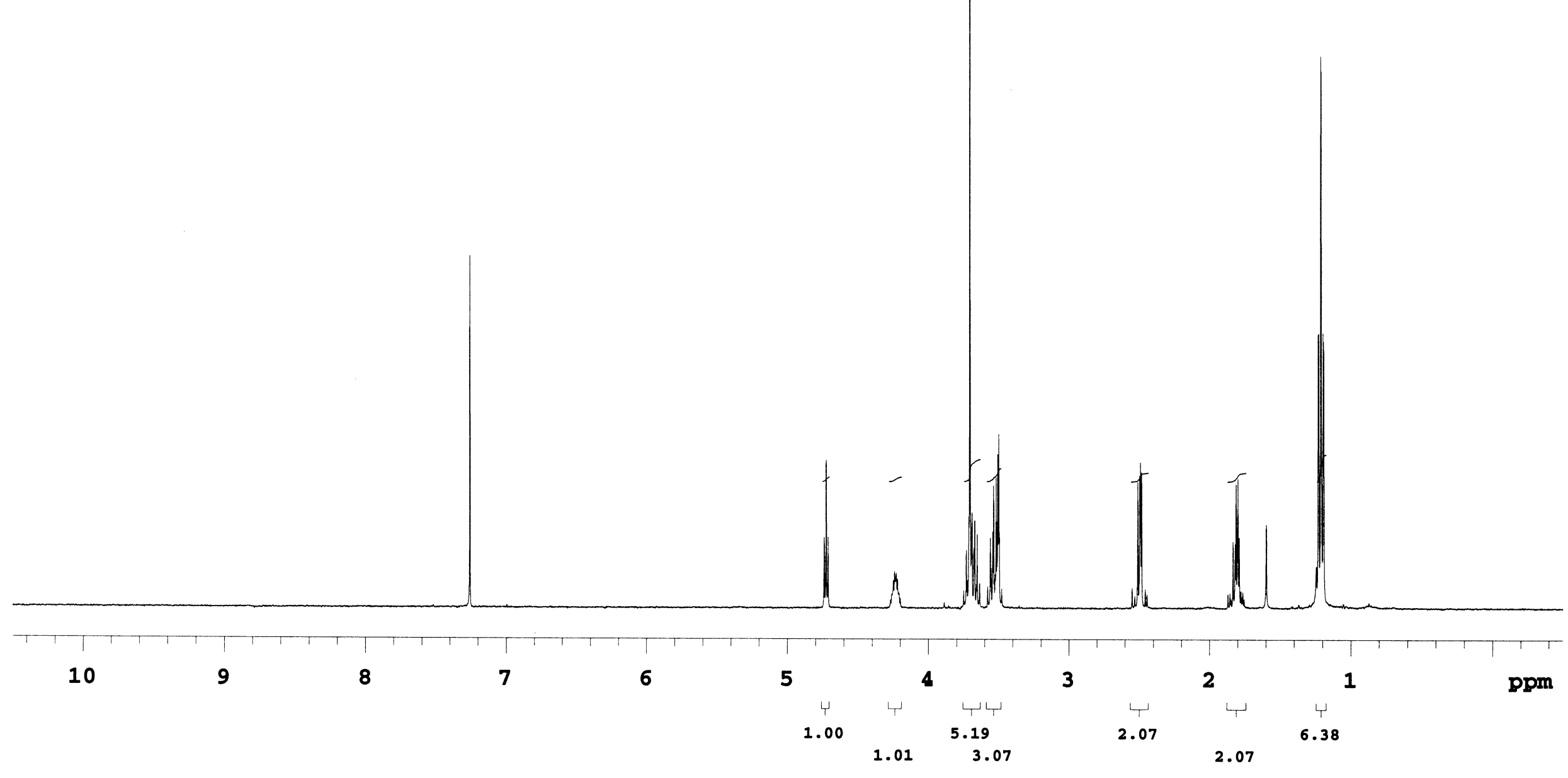




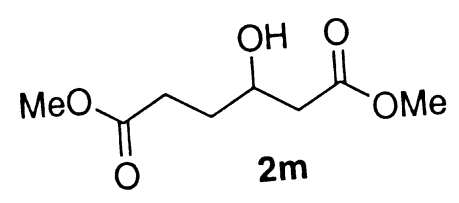




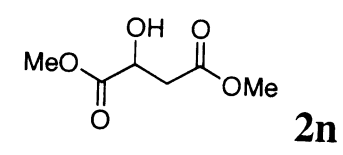

$2 n$

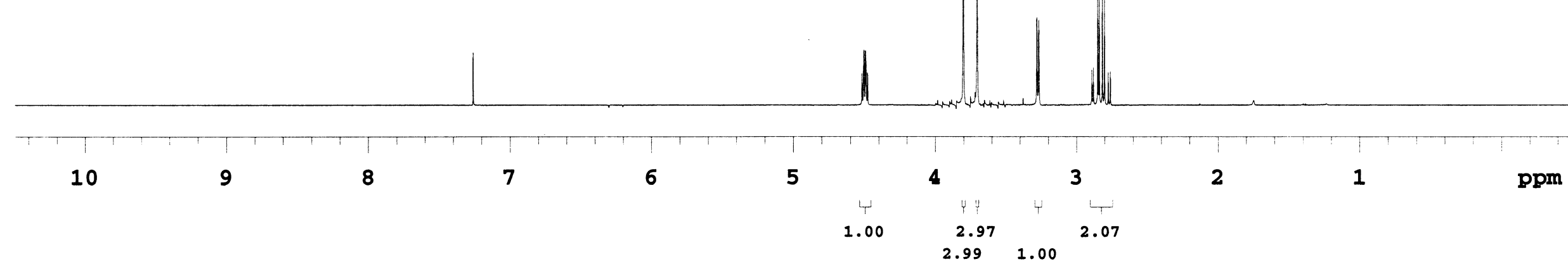




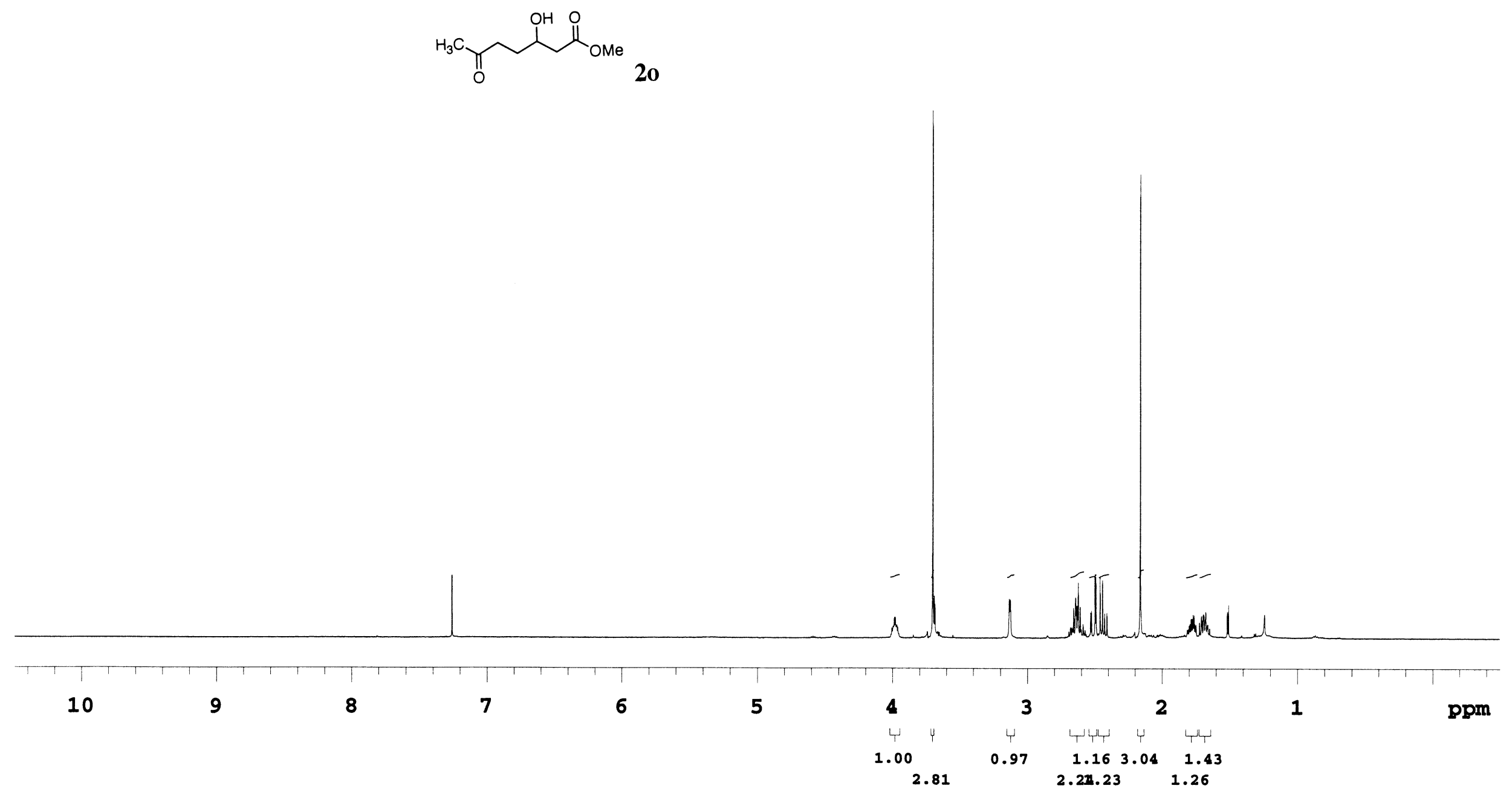




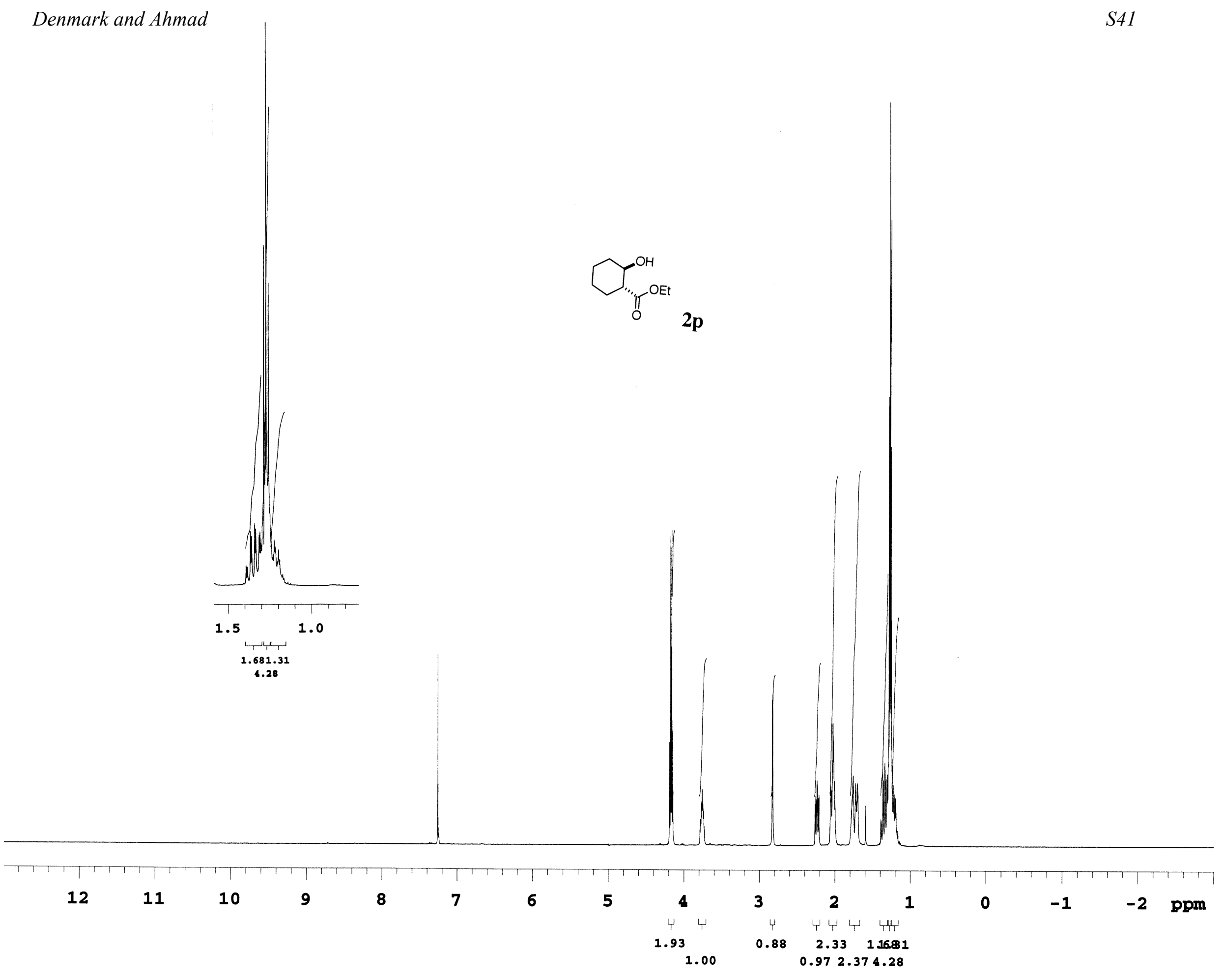



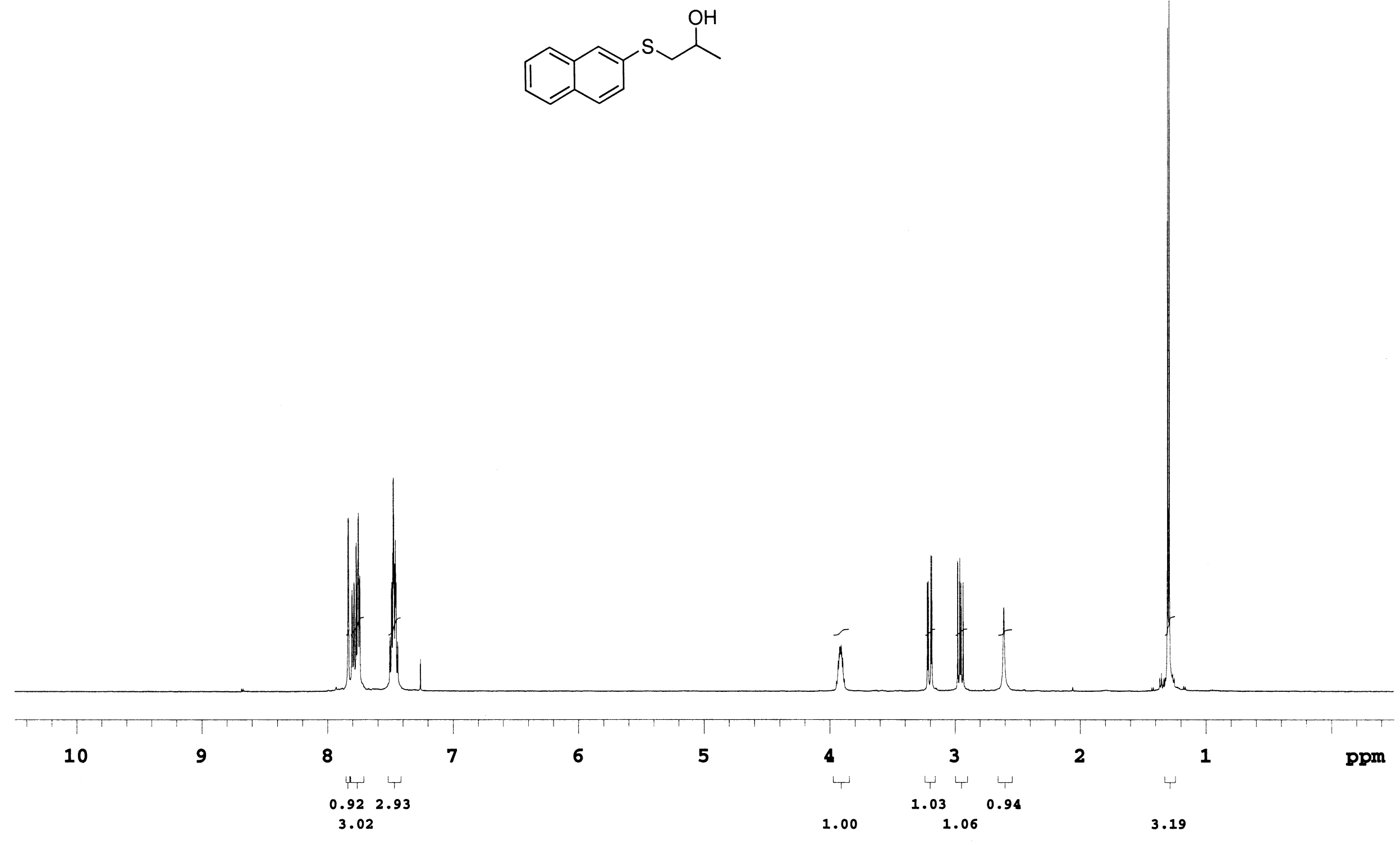



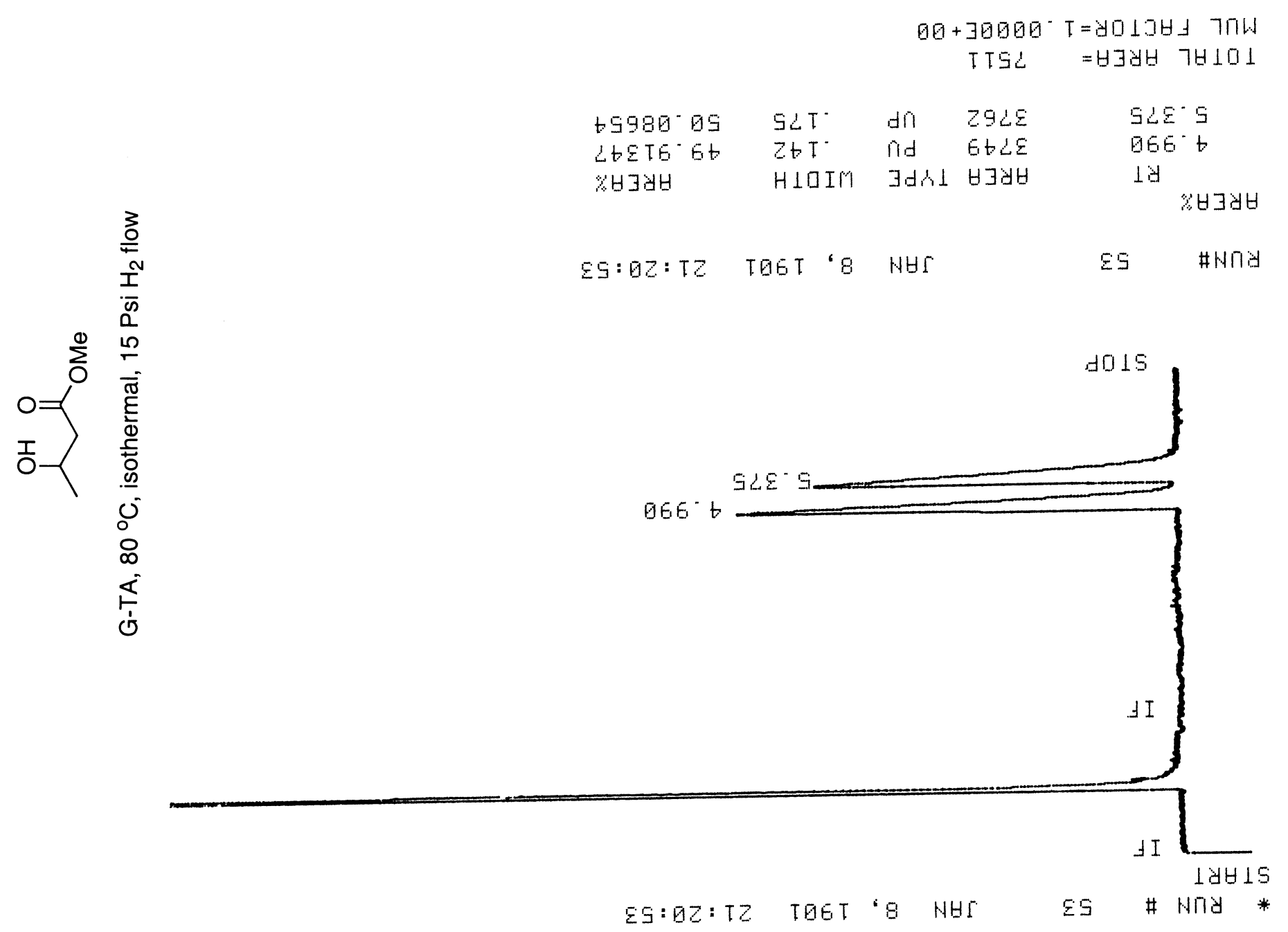




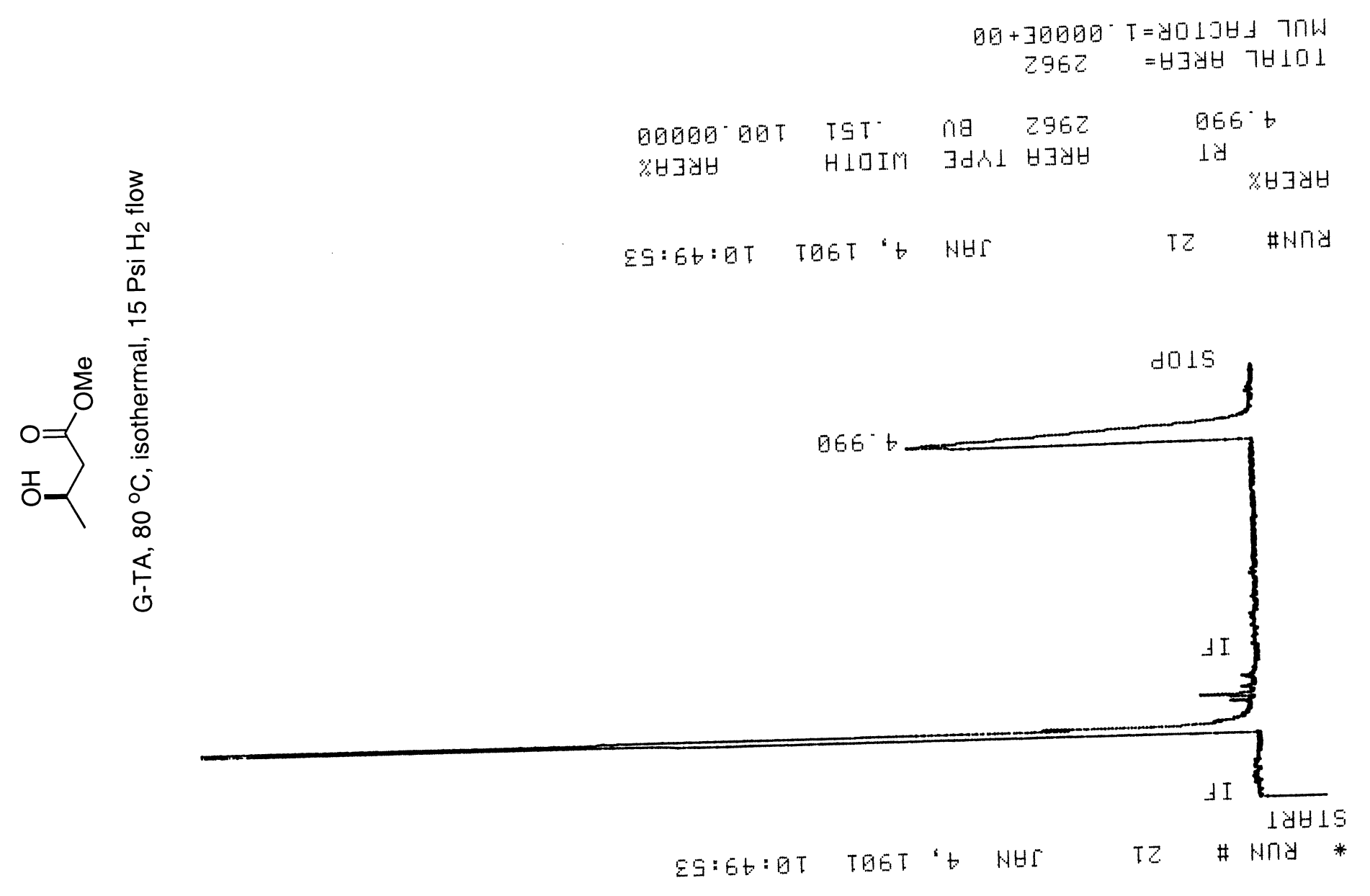

